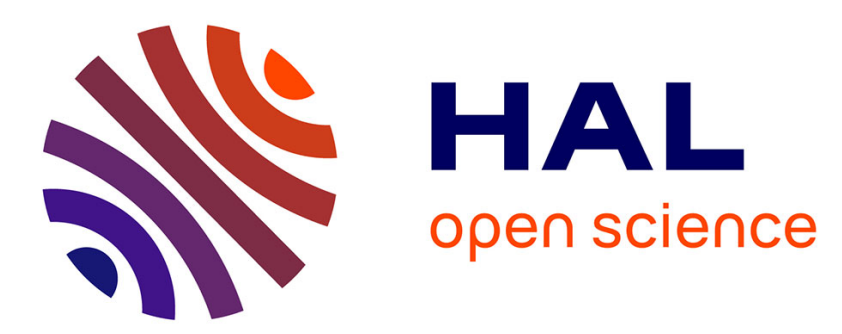

\title{
Using discrete systems to exhaustively characterize the dynamics of an integrated ecosystem
}

Cedric Gaucherel, Franck Pommereau

\section{To cite this version:}

Cedric Gaucherel, Franck Pommereau. Using discrete systems to exhaustively characterize the dynamics of an integrated ecosystem. Methods in Ecology and Evolution, 2019, 10 (9), pp.1615-1627. 10.1111/2041-210X.13242 . hal-02265554

\section{HAL Id: hal-02265554 \\ https://hal.science/hal-02265554}

Submitted on 11 Oct 2019

HAL is a multi-disciplinary open access archive for the deposit and dissemination of scientific research documents, whether they are published or not. The documents may come from teaching and research institutions in France or abroad, or from public or private research centers.
L'archive ouverte pluridisciplinaire HAL, est destinée au dépôt et à la diffusion de documents scientifiques de niveau recherche, publiés ou non, émanant des établissements d'enseignement et de recherche français ou étrangers, des laboratoires publics ou privés. 
(1)

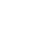

\title{
Using discrete systems to exhaustively characterize the dynamics of an integrated ecosystem
}

\author{
Gaucherel C. ${ }^{(1)}{ }^{*}$, Pommereau F. ${ }^{(2)}$ \\ ${ }^{1}$ AMAP - INRA, CIRAD, CNRS, IRD, Université Montpellier, Montpellier, France \\ ${ }^{2}$ IBISC, Université d’Evry-val d'Essonne, Université Paris-Saclay 91025 Evry, France \\ Correspondence to: \\ * Cédric Gaucherel \\ INRA - EFPA, UMR AMAP, TA A.51/PS2, 34398 Montpellier Cedex 5 (France) \\ Tel.: $33(0) 467616586$ \\ Fax: $33(0) 467615668$ \\ Email: gaucherel@cirad.fr
}

Word count: 6352 Running head: Ecosystem dynamics viewed by Petri nets.

Published as: Gaucherel, C, Pommereau, F. Using discrete systems to exhaustively characterize the dynamics of an integrated ecosystem. Methods Ecol Evol. 2019; 10: 1615- 1627.

https://doi.org/10.1111/2041-210X.13242 
1. To understand long-term ecosystem dynamics, several concepts have recently been proposed that consider 'basins of attraction' used to express resilience, and 'tipping points' that express sharp change in an ecosystem's behavior. However, these temporal features remain difficult to identify and quantify, because current models usually only focus on a part of the whole ecosystem behavior, whereas a holistic approach should be preferred.

2. We propose an original family of models based on discrete systems and designed to comprehensively characterize ecosystem dynamics holistically and over the long term. We developed a qualitative model based on Petri nets, made up of a relational graph (interaction network) that was then rigorously handled using transition rules. Unlike traditional modelling and graph theory approaches, transition rules can strongly modify the graph structure (i.e. a dynamic topology occurs).

3. We examined the value of Petri nets when applied to the simple ecosystem of a termite colony. A termite colony comprises abiotic and biotic components and processes that we explored along all of their possible trajectories.

4. Several temporal features were easily detected and quantified, such as basins of attraction (i.e. strongly connected states), tipping points (critical transitions along trajectories) and various kinds of collapses (functioning systems whose structures were nevertheless fixed). We propose that Petri nets developed for more complex ecosystems will provide original insights into their holistic behavior.

Keywords: ecosystem resilience; ecosystem collapse; integrated model; discrete systems; formalization. 


\section{Introduction}

The concept of resilience is used to describe an ecosystem when it persists in the face of perturbations (Holling 1973; DeAngelis 1980; Scheffer 2009). This concept is the subject of continued debate, with several ongoing definitions, although most ecologists acknowledge its relevance to understanding ecosystem dynamics. While easily understandable, the concept of resilience remains difficult to quantify and predict (Walker et al. 2004; Hirota et al. 2011; Karssenberg, Bierkens \& Rietkerk 2017). Recently, a conceptual view has been put forward to represent the richness of ecosystem dynamics. This approach proposes to plot the parameter zones in which the system is considered to be resilient, as 'basins of attraction' or 'wells,' whose depth is said to quantify the intensity of resilience (Scheffer et al. 2001; Scheffer et al. 2015). It is now necessary to develop the most appropriate methods to determine the behavior of these basins of attraction, therefore a new type of model needs to be developed for this purpose.

An ecosystem can possess several potential basins between which it can alternate (Thom 1975; Gaucherel 2010). Consequently, the system possesses a range of possible trajectories, within or between these basins, that are considered relatively stable zones, i.e., where the system is more frequently found. When the system shifts from one basin to another, it is said to cross a tipping point (TP) or a catastrophic shift (Scheffer et al. 2001; Hirota et al. 2011). Several studies have shown that ecosystems and environmental systems experience sharp TPs (Scheffer et al. 1993; Carpenter 2003), over the short and/or long term (Hély et al. 2009; Lenton et al. 2012). However, TPs, like the resilience to which they are connected, are notoriously difficult to identify and quantify. It is therefore critical to correctly identify: i) the basins of attraction of an ecosystem, ii) basin depth, and iii) the relative locations of these basins in the potential landscape, if we are to deduce the possible TPs linking basins to each other (Walker et al. 2004; Scheffer et al. 2015).

Several models have been proposed to describe resilience and tipping points in various systems. The pioneering approach in this field was the catastrophe theory developed by (Thom 1975). Thom 
(1975) created powerful yet hard-to-handle algebraic tools to analyze and understand the potential of any system. In ecology, the common model used is usually a one dimensional ordinary differential equation handling the central state-variable (e.g. biomass, Scheffer et al. 2001; Van Nes \& Scheffer 2007). The direct consequences of using such equations are that they focus on the dominant behaviour in a system and reduce the study to a fixed phase-space defined by the state-variables. Conversely, more rare trajectories can occur, and most catastrophes may even require a qualitative shift in the system variables as well as in the phase-space (Thom 1975; Hély et al. 2019). For example, a shift from a forest to a grassland state will drastically change its vegetation composition, fire regime, faunal communities and trophic network (Hély et al. 2009; Hély et al. 2019). Tipping points will disrupt the entire system structure and functioning, and therefore should be examined using models dedicated to exhaustive behavior analysis.

To model a rapidly changing ecosystem, we developed a method using Petri nets, which have been used successfully in theoretical computer science and systems biology (Pommereau 2010; Reisig 2013). Petri nets are similar to Boolean networks (Kauffman 1969; Thomas \& Kaufman 2001), as they are well adapted to formalize network topological changes. To our knowledge, such discrete and qualitative models have rarely been used in ecology (but see Ewing et al. 2002; Cordier, Largouët \& Zhao 2014; Baldan et al. 2015; Gaucherel et al. 2017). Among discrete models, Petri nets are powerful tools that enable the rigorous formalization of exhaustive changes in an ecosystem's structure. Petri nets can be used to scrutinize all possible fates of a system and to map every potential qualitative trajectory. Although qualitative, it is nevertheless possible to compute quantitative metrics from the model outputs and deduce many system properties (Koch, Reisig \& Schreiber 2011).

We examine the potential of Petri nets to model a simple ecosystem. Our objective is to manipulate an ecosystem-like structure, composed of biotic and abiotic (and anthropogenic-like) components working in close interaction, with potential disturbances occurring (Turner 2009; Gaucherel et al. 
2017). Therefore, we chose to model a (Macrotermitinae) termite colony, because a full termite colony develops from reproductives (queen and king) and external resources, and can drastically shift into a different system when experiencing strong disturbance, e.g., mound destruction or attack. The termite colony could then reach a collapsed (or deadlock) state via various trajectories. We need a rigorous, formalized model to confidently identify resilience zones, such as basins of attraction, the tipping points separating them, and possibly other properties of system behavior such as collapse.

\section{Materials and methods}

\section{Case study - a eusocial insect ecosystem}

Eusociality is characterized by cooperative brood care, overlapping adult generations, and division of labor between castes that leads to reproductive and sterile groups (Costa \& Fitzgerald 2005). We chose to model eusocial insect colonies for their propensity to experience drastic changes (tipping points) over time, but any other ecosystem-like models may be used. We chose to work on Macrotermitinae termites, which construct large colonies (up to millions of inhabitants, Fowler et al. 1986), (Turner 2009) . Termites cultivate fungi in special chambers, build aerial structures (called mounds) to improve air circulation, and divide each nest into a royal chamber, fungus chambers, and egg rooms (Fig. 1a, Suppl. Mat. Appendix 1). Given the ability for this eusocial species to produce fungi, termites might also be considered as mimicking agricultural (farmer) activities. One way of conceptualizing the ecosystem under study is to represent it as a network of elements in interaction (Fig. 1b). This ecosystem graph is then handled by the following model.

\section{Petri nets}

We propose a twofold approach: i) first, the interaction network of the ecosystem is represented by a graph designed to gather any kinds of ecological processes (not to be confused with purely trophic, 
pollinator or parasitic networks, Thébault \& Fontaine 2010; Campbell et al. 2011); ii) second, a rigorous model based on a Petri net is developed to formalize any change in the graph topology (i.e. neighboring relationships). Petri nets, developed in computer sciences (Pommereau 2010; Reisig 2013) and also used in systems biology (Murata 1989; Blätke, Heiner \& Marwan 2011; Koch, Reisig \& Schreiber 2011), are powerful tools for rigorously formalizing changes in network topologies occurring during the system dynamics. The two major differences between a Petri Net model and other network models used in ecology (Thébault \& Fontaine 2010; Kéfi et al. 2016) are that Petri net models deal with topological changes during simulations and can explore all possible dynamics of the system. Although the maximal interaction network remains predefined in our study, it is continuously modified within runs and any drastic changes are fully controlled (Gaucherel et al. 2017). These differences therefore make our model difficult to compare to existing models. For example, a predator-prey model would focus only on one ecosystem state of the dynamics produced. Such discrete models provide convenient and commonly used tools to exhaustively analyze the states of the system (called the state-space), the relatively stable (resilient) zones, the tipping points and any system trajectory (Walker et al. 2004; Scheffer et al. 2015). In this section, we start by presenting a simplistic predator-prey model and we then define multisets required to rigorously define Petri nets that will then be used to model the termite colony.

\section{A simplistic predator-prey Petri net}

137 We first illustrate Petri nets with a simplistic but unrealistic predator-prey system. In the predatorprey system, only two nodes are defined as the prey and predator populations (these are not individuals, Fig. 2a). The state of the system is defined by the set of "+" and "-" nodes, graphically drawn as having a token (a mark) or not, representing the presence and absence of each system component, respectively (see next section for a formal definition). The maximal number of possible system states is $2^{\# \text { nodes }}$ and grows exponentially with the node number. The state of a node depends on the node states to which it is connected, while a connection between nodes exists as soon as one 144 process makes this connection explicit (Fig. 2b). The rules correspond to any physicochemical, 
biological and ecological and/or human-like processes (or sub-processes), and thus represent all interactions between nodes composing the ecosystem studied. In the predator-prey system, only two rules are defined: R1, the predation itself, and R2, the mortality (Fig. 2b).

In the formalism of this ecosystem, a rule is made up of condition and realization parts written by convention as: "transition's name: condition $\rightarrow$ realization". In the predator-prey system, the rules are written as $\mathrm{R} 1: \mathrm{P}+, \mathrm{N}+\rightarrow \mathrm{N}-$ and as $\mathrm{R} 2: \mathrm{N}-, \mathrm{P}+\rightarrow \mathrm{P}_{-}$, with $\mathrm{P}$ and $\mathrm{N}$ the predator and prey populations, respectively. Since the rules modify node states, they also change the system state. Therefore, the system will shift from one state to another one through the successive applications of rules (Fig. 2b). These rules can then be translated into a Petri net in which the nodes are translated to pairs of places, and the rules are translated to transitions, both being connected through oriented $\operatorname{arcs}$ (Fig. 2c). Such a Petri net has its own dynamics: a transition that has enough tokens in its input places (those for which an arc exists from the input place to the transition) may fire (trigger), consuming tokens in its input places and producing new tokens in its output places (those for which an arc exists from the transition to the input place). These dynamics of the Petri net produce the state space, which provides the set of all system states reachable by the rules defined (Fig. 2d). As a corollary, the states are also connected to each other by some of these rules in the state space. The size of the state space is usually much smaller than the number of possible system states, because the computation starts from a specific initial condition and because rules have specific application conditions.

\section{Formal definition of Petri nets}

We now give a rigorous definition of Petri nets. To this end, we first need to define a multiset over a set $X$ as a function $\mu: X \rightarrow \mathbb{N}$. We denote by $X^{*}$ the set of all the multisets over $X$. A multiset $\mu \in X^{*}$ can be extended to a larger set $Y \supset X$ by defining $\mu(y) \stackrel{\text { def }}{=} 0$ for all $y \in Y \backslash X$. Given $\mu_{1}$ and $\mu_{2}$ in $X^{*}$, we define:

\footnotetext{
- the sum of $\mu_{1}$ and $\mu_{2}$ by $\left(\mu_{1}+\mu_{2}\right)(x) \stackrel{\text { def }}{=} \mu_{1}(x)+\mu_{2}(x)$ for all $x \in X$;
} 
- the comparison of $\mu_{1}$ and $\mu_{2}$ by $\mu_{1} \leq \mu_{2}$ iff $\mu_{1}(x) \leq \mu_{2}(x)$ for all $x \in X$;

- the difference of $\mu_{1}$ and $\mu_{2}$ by $\left(\mu_{1}-\mu_{2}\right)(x) \stackrel{\text { def }}{=} \max \left(0, \mu_{1}(x)-\mu_{2}(x)\right)$ for all $x \in X$;

- $\quad$ the product by $k \in \mathbb{N}$ by $(k \times \mu)(x) \stackrel{\text { def }}{=} k \times \mu(x)$ for all $x \in X$.

Multisets may be denoted in extended sets notation as $\mu=\{a, a, b\}$ that is such that $\mu(a)=2$, $\mu(b)=1$ and $\mu(c)=0$ for all $c \notin\{a, b\}$. A subset of a set $X$ may be considered as a multiset by identifying it to its characteristic function.

A marked Petri net is a tuple $N \stackrel{\text { def }}{=}(S, T, W, M)$ such that:

- $\quad S$ is the finite set of places;

- $T$, disjoint from $S$, is the finite set of transitions;

- $W:(S \times T) \cup(T \times S) \rightarrow \mathbb{N}$ is the weight function that defines arcs;

- $M \in S^{*}$ is the marking, a multiset of places representing the state of the Petri net (the number of tokens stored in each place).

Petri nets are commonly depicted as graphs (Fig. 2c), whose nodes are places (depicted as roundshaped nodes) and transitions (square-shaped nodes), and whose edges are the arcs connecting them with non-zero weight (i.e., an arc drawn as $x \rightarrow y$ is labeled by $W(x, y)>0$, with 1 usually omitted if $W(x, y)=1$ ), and the marking is depicted as black tokens $\bullet$ within places (or the number of tokens when this is more readable). An arc from a place to a transition (or conversely from a transition to a place) is called an input (conversely output) arc (Fig. 2c). A pair of input/output arcs with the same weights and between the same place and transition may be replaced by a single arc with one arrow at each end (like the arcs connected to $\mathrm{Ac}^{+}$in Fig. 3b).

For every transition $t \in T$, we define $\stackrel{\overleftarrow{t} \stackrel{\text { def }}{=}}{=} \sum_{s \in S} W(s, t) \times\{s\} \in S^{*}$ and $\stackrel{\vec{t}}{=} \stackrel{\text { def }}{=} \sum_{s \in S} W(t, s) \times\{s\} \in S^{*}$. For instance, we have $\overleftarrow{R 1}=\{N+\}$, and $\overrightarrow{R 1}=\{P+, N-\}$ in Fig. 2c.

Let $N \stackrel{\text { def }}{=}(S, T, W, M)$ be a marked Petri net. A transition $t \in T$ is enabled at marking $M$ iff $t \leq M$. In such a case, $t$ may be fired yielding a new marking $M^{\prime} \stackrel{\text { def }}{=} M-\overleftarrow{t}+\vec{t}$, which is denoted by $M\left[t>M^{\prime}\right.$. 
The marking graph (also called the reachability graph) of $N$ is the smallest graph $[N\rangle \stackrel{\text { def }}{=}(V, E)$ such that $M \in V$ and for all $M^{\prime} \in V$ such that $M^{\prime}[t\rangle M^{\prime \prime}$ for a transition $t \in T$, then we also have $M^{\prime \prime} \in V$ and $\left(M^{\prime}, t, M^{\prime \prime}\right) \in E$. Here, $V$ stands for vertices (nodes) and $E$ for edges. As an example, the marking graph of the predator-prey Petri net depicted in Fig. $2 \mathrm{a}$ is shown in Fig. $2 \mathrm{~d}$.

Essentially, a Petri net models the possible states of a system and the possible transitions between them, while the Petri net marking additionally uses tokens to indicate which state the system is currently in.

\section{Results}

\section{Formal definition of a termite colony ecosystem}

We modelled a termite colony as an ecosystem made up of biotic and abiotic components. Therefore, we defined an interaction network (i.e. a relational graph between system components) connecting nodes of various natures, with edges modelling various interactions and processes (Fig. 1b, Appendix 1). A system node could belong to any one of the five categories: the permanent environment outside the colony (plotted in orange), resources (green), spatial structures (violet), inhabitants (blue), and competitors/predators (black) (Figure 1). A node could only take either one or the other of two Boolean states (Gaucherel et al. 2017): On or + (presence of the node in the ecosystem) and Off or - (node is absent). We modelled Boolean values through pairs of complementary places, which is the usual way to work with the absence of test-to-zero in Petri nets (as a transition cannot test for the absence of a token). For termite ecosystems, an extended graph made up of 12 nodes was defined as the best compromise between over and under-simplified descriptions (Table 1). 
The formal specification of such an ecosystem consists in a triplet $\left(\mathbb{E}, s_{0}, R\right)$ such that $\mathbb{E}$ is a set of entities, $s_{0} \subseteq \mathbb{E}$ is the initial state, and $R$ is a set of reaction rules (a process may fire several rules and transitions). Entities can be declared with their respective names and initial states (Table 1$)$. The initial state $s_{0}$ is the set of all the entities declared as initially 'On'. Here, we chose $s_{0} \stackrel{\text { def }}{=}$ $\{R p, S l, A t, A c\}$, representing reproductives, soil, atmosphere and competitors, respectively. A state of such a system is defined, like the initial state, by a subset of $\mathbb{E}$. For instance, having $S_{0} \stackrel{\text { def }}{=}$ $\{R p, S l, A t, A c\}$ as above means that every entity in $s_{0}$ is present $(+)$ in this system state and, consequently, every entity in $\mathbb{E} \backslash s_{0}$ is absent (-) from this system state.

The dynamics of the system is described by a set of reaction rules of the form $\left(\alpha^{+}, \alpha^{-}, \omega^{+}, \omega^{-}\right) \subseteq \mathbb{E}^{4}$ such that $\alpha^{+} \cap \alpha^{-}=\omega^{+} \cap \omega^{-}=\emptyset$. For convenience, the rules may be numbered and denoted as $n: \alpha \rightarrow \omega$ where $n$ is the identifying number, $\alpha$ is the list of entities in $\alpha^{+}$followed by a + sign, plus the list of entities in $\alpha^{-}$followed by a - sign, and $\omega$ is similarly defined with $\omega^{+}$and $\omega^{-}$. For example, rule 12 in Table 2 is $(\{A c\},\{S d\}, \emptyset,\{W k, R p\})$ and is more conveniently written as $12: A c^{+}, S d^{-} \rightarrow$ $W k^{-}, R p^{-}$as in rewriting systems (Lindenmayer 1978; Gaucherel et al. 2012). This rule specifies that if $A c$ is present and $S d$ is absent, then the system may evolve by switching $W k$ and $R p$ to the 'absent' state (regardless of their current states, Fig. 3a). For example, translated into ecological terms, rule number 12 means that if ant competitors are present when termite soldiers are absent, then termite workers and reproductives can be killed (i.e. if the rule is applied).

A rule $r \stackrel{\text { def }}{=}\left(\alpha^{+}, \alpha^{-}, \omega^{+}, \omega^{-}\right)$is enabled at a state $s \in \mathbb{E}$ iff $\left(\alpha^{+} \subseteq s\right) \wedge\left(\alpha^{-} \cap s=\emptyset\right)$; in such a case, $r$ may be fired, yielding a new state $s^{\prime} \stackrel{\text { def }}{=}\left(s \backslash \omega^{-}\right) \cup \omega^{+}$, which is denoted by $s \stackrel{r}{\rightarrow} s^{\prime}$. We ignore firings that do not change the state (so-called self-loops), for instance, applying Rule 9 when $F g$ and $S d$ are already absent does not bring new information (Table 2). The state space of a model is the smallest we also have $s^{\prime} \in E$ and there is an edge $\left(s, r, s^{\prime}\right) \in V$. 
241 By defining the state-space from a set of reaction rules, we have provided its semantics, which is the

242 definition of its behavior. Hence, the reaction rules are just syntactic artefacts; by defining their 243 semantics, they become the specification of a behavior. Similarly, marking graphs are the semantics 244 of Petri nets. In this section, we provide another semantics for reaction rules in terms of a translation to Petri nets. While the state-space semantics is useful to understand systems at a high level of abstraction, it is not convenient for automated analysis since no tool exists for reaction rules. The Petri net semantics is a convenient solution to enable the use of the numerous existing theoretical and software tools to analyze Petri nets. Crucially, at the end of the section, we demonstrate that both semantics are equivalent (and see Appendix 2), which is a common way of proceeding (Best, Devillers \& Koutny 2001). The Petri net semantics of such systems is computed in two successive steps. First, (ecological) rules are normalized to make them simpler and unambiguous, then a Petri net is computed.

The need for normalization arises from the structure of reaction rules that do not enforce the leftand right-hand sides of rules to involve exactly the same entities. To illustrate the need for normalization, consider rule $12: A c^{+}, S d^{-} \rightarrow W k^{-}, R p^{-}$given above (Fig. 3a). This rule has two ambiguities: i) it may be applied regardless of the current state of $W k$ and $R p$, which corresponds to four distinct situations; and ii) it is not complete as it does not specify what happens to $A c$ and $S d$ after the rule is fired. So, an ecological process may often be considered as a meta-rule in the Petri net, and the normalization procedure aims at producing unambiguous and complete (exhaustive) transitions from the meta-rules provided in the specification.

For this purpose, we apply two transformations corresponding to the two cases above. Consider a rule $r \stackrel{\text { def }}{=}\left(\alpha^{+}, \alpha^{-}, \omega^{+}, \omega^{-}\right)$, then take $\alpha \stackrel{\text { def }}{=} \alpha^{+} \cup \alpha^{-}, \omega \stackrel{\text { def }}{=} \omega^{+} \cup \omega^{-}$, and $\chi \stackrel{\text { def }}{=} \omega \backslash \alpha, r$ is replaced by the set of rules $R_{r} \stackrel{\text { def }}{=}\left\{\left(\alpha^{+} \cup x, \alpha^{-} \cup(\chi \backslash x), \omega^{+}, \omega^{-}\right) \mid x \in 2^{\chi}\right\}$. Intuitively, the left-hand side of $r$ 
is augmented in every possible manner so that it becomes a partition of the entities involved in $r$, i.e., those from $\alpha \cup \omega$. Note that the new rules are still somewhat ambiguous because their left-hand side still lacks the entities that are not involved in the original rule, but this will be neatly handled in the Petri net semantics. Then, the right-hand sides of the new rules are augmented with the entities that appear in $\alpha$ but not in $\omega$ so that they are left untouched when the rule is fired, i.e. a change that is not explicitly specified should not occur, which is consistent with the semantics defined above in which $\alpha$ is not involved in the computation of successor states. So the normalized set of rules originated from $r$ is $\left\{\left(\alpha^{+}, \alpha^{-}, \omega^{+} \cup\left(\alpha^{+} \backslash \omega\right), \omega^{-} \cup\left(\alpha^{-} \backslash \omega\right)\right) \mid\left(\alpha^{+}, \alpha^{-}, \omega^{+}, \omega^{-}\right) \in R_{r}\right\}$.

For example, rule $12: A c^{+}, S d^{-} \rightarrow W k^{-}, R p^{-}$is first replaced with the four new rules below by completing its left-hand side (Fig. 3b):

$$
\text { - } \quad A c^{+}, S d^{-}, W k^{-}, R p^{-} \rightarrow W k^{-}, R p^{-}
$$

- $\quad A c^{+}, S d^{-}, W k^{-}, R p^{+} \rightarrow W k^{-}, R p^{-}$

- $\quad A c^{+}, S d^{-}, W k^{+}, R p^{-} \rightarrow W k^{-}, R p^{-}$

- $\quad A c^{+}, S d^{-}, W k^{+}, R p^{+} \rightarrow W k^{-}, R p^{-}$

Then, the right-hand sides of these rules are completed in order to preserve $A c$ and $S d$ :

- $\quad A c^{+}, S d^{-}, W k^{-}, R p^{-} \rightarrow W k^{-}, R p^{-}, A c^{+}, S d^{-}$

- $\quad A c^{+}, S d^{-}, W k^{-}, R p^{+} \rightarrow W k^{-}, R p^{-}, A c^{+}, S d^{-}$

- $\quad A c^{+}, S d^{-}, W k^{+}, R p^{-} \rightarrow W k^{-}, R p^{-}, A c^{+}, S d^{-}$

- $\quad A c^{+}, S d^{-}, W k^{+}, R p^{+} \rightarrow W k^{-}, R p^{-}, A c^{+}, S d^{-}$

\section{Translation into Petri nets}

The normalized rules can be easily translated into Petri nets in two steps: i) for each entity $e \in \mathbb{E}$, two places are created: $e^{+}$and $e^{-}$to represent the present and absent states of the entity respectively. The rule that corresponds to the initial state given in the specification is marked with one token, the 
other place is left empty (no token); ii) for each normalized rule in order to preserve Wk and Te: $r \stackrel{\text { def }}{=}$ $\left(\alpha^{+}, \alpha^{-}, \omega^{+}, \omega^{-}\right)$, a transition $t_{r}$ is added with arcs such that:

- $W\left(e^{+}, t_{r}\right) \stackrel{\text { def }}{=} 1$ for all $e \in \alpha^{+}$,

- $W\left(e^{-}, t_{r}\right) \stackrel{\text { def }}{=} 1$ for all $e \in \alpha^{-}$,

- $W\left(t_{r}, e^{+}\right) \stackrel{\text { def }}{=} 1$ for all $e \in \omega^{+}$,

- $W\left(t_{r}, e^{-}\right) \stackrel{\text { def }}{=} 1$ for all $e \in \omega^{-}$,

- $W\left(t_{r}, p\right) \stackrel{\text { def }}{=} 0$ for any other place $p$.

The last item above shows how we handle the entities that are not at all involved in a rule.

Moreover, in order to eliminate self-loops here too, we ignore transitions that fire but do not change

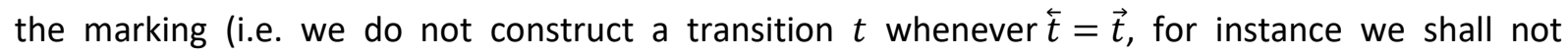
construct the top-most transition in Fig. 3b).

At this stage, we demonstrate a theorem that proves a strong equivalence between the reaction rules and their translation into a Petri net (Appendix 2). In general terms, we prove that the statespace built from the reaction rules is strongly similar to the marking graph of the Petri net obtained through the translation of reaction rules into Petri nets (Best, Devillers \& Koutny 2001). Both graphs are isomorphic (i.e. they are identical, yet with labeling that needs to be translated from one graph to the other). This property is both usual and essential in computer sciences when several layers of formalism are handled. A precise relationship exists between the two formalisms and their semantics (Appendix 2, Fig. S1) and the theorem provides a mathematical guarantee that we can study the marking graph instead of the state-space of the original system.

Theorem 1: Let $\left(\mathbb{E}, s_{0}, R\right)$ be a reaction rules model and $(S, T, W, M)$ be its Petri net semantics. Then, the state space of $\left(\mathbb{E}, s_{0}, R\right)$ is isomorphic to the marking graph of $(S, T, W, M)$ (see the proof in Appendix 2). 
313 The Petri net of the termite ecosystem is made of 12 ecological components (i.e. nodes, Table 1, Fig.

314 1b), and 15 ecological processes (i.e. rules, Table 2). The exact Petri net obtained quickly becomes unreadable (i.e. too many nodes and edges), as the system becomes more complex, but there is no need to draw it to analyze the system. Rather, as a first analytical step, we can compute its marking graph (theorem 1). Modelling of the termite colony reaches only 109 states (among $2^{12}$ possible states), so that we can draw the exhaustive state-space to visualize it (Fig. 4a). For larger systems, the analysis can be performed automatically and without drawing the state space too. The state-space graph obtained here comprises several (colored) elements which we will further describe and interpret in ecological terms: the initial state (numbered 0, and drawn as a hexagon, Fig. 4a-A), two topological structures usually called strongly connected components (Fig. 4a-B and 4a-B'), some decisive paths (e.g. ecosystem trajectories and tipping points, Fig. 4a-C), ultimately leading upward to some basins and their associated deadlocks (i.e. states from which no other states are reachable, Fig. 4a-D and 4a-D', squares).

Any strongly connected component (SCC) is defined as a set of system states (nodes of the state space) in which every state may be reached from any other state of the SCC. As such, SCCs are important as they allow the termite ecosystem to run indefinitely, in a kind of dynamic (structural) equilibrium (see Discussion). From the initial state 0 , the system may rapidly evolve to deadlock 2 , always through the execution of rule 12 corresponding to the death of reproductives in the presence of ant competitors (Fig. 3). Otherwise, the system reaches SCC B (Fig. 4a-B, orange color) when it fires rules 5, 6 , and then 3 . So, as a first observation, the system can stay "alive" only if it successively executes these three rules. If the system stays alive, it reaches the large SCC B. From SCC B, the system may reach the second large SCC B' (Fig. 4a-B', green). From both SCCs, the system may exit towards two deadlocks at the left and right hands of the state space, respectively (Fig. 4a-D and 4a$\left.D^{\prime}\right)$. Deadlocks correspond to states in which the system can no longer evolve, which, in our case, 
corresponds to ecosystem collapses (i.e. they contain no more termites and cannot recover from any previous state).

\section{Strongly connected components}

The termite system exhibits two large SCCs labeled B and B' (Fig. 4a-B and 4a-B'), within which the colony may circulate forever. Here, we propose that any SCC is a kind of (qualitative and structural) stability which behaves like a basin (not to be confused with a "basin of attraction") where it is possible to stay or occasionally leave. When considered statistically, such SCCs channel the ecosystem through states which are more numerous for larger basins and take longer to leave for trajectories further away from the SCC exit. Although the model is qualitative, the number of states in the SCC and the maximal distance to its exit appear as relevant proxies to quantify the associated basin.

From each state of SCC B where $S d$ is present, we can fire rule 11 (soldiers kill ants, Table 2) to reach a state of SCC B' (but not conversely) that is exactly the same, except that competitors $(A C)$ have switched to the 'absent' state (both SCCs are isomorphic). This has been checked programmatically on the state space. However, from every state in SCC B where $S d$ is absent, we can fire rule 12 (ants kill termites) or rule 13 (the absence of fresh air kills termites) and leave the SCC in a deadlock (and its basin). Considering this isomorphism, we can concentrate on SCC B' only, and zoom in on it by hiding states outside the SCC (Fig. S3a). The higher the distance to exit (in steps) the SCC, the less likely the system leaves the SCC and reaches another basin containing a deadlock leading to the inevitable (structural) collapse of the system. A state labeled with a null exit distance does not necessarily mean that the system will exit, rather that it may exit into a single move. By more finely studying the SCC B' graph, it is possible to list many other observations on the termite system behavior (see Appendix 3 and Fig. S3 for a refined analysis). 
361

362

363

To focus on system trajectory instead of system states, it is possible to compute the marking graph in which SCCs have been reduced to a single node (also called the SCC graph or merged state space, Fig. 4b). When not reduced, this trajectory graph may rapidly become unreadable. It is possible to automatically (via programming) simplify this SCC graph by merging basins to a single node including the deadlock they lead to (Fig. 4b). From this convenient reduction of the ecosystem trajectories, it becomes easy to identify specific and contrasting paths leading to the ecosystem collapse (Fig. 4b, squares), and the tipping points separating them (e.g. Fig. 4a, red segment, see Appendix 3 for a refined analysis of trajectories).

\section{Discussion}

A goal of ecology is to develop strategies and tools for mapping an ecosystem's long-term dynamics, as well as some dynamical properties such as basins and sharp transitions (e.g. tipping points, TPs). This issue is a real challenge and with current models it can be hard to quantify the resilience and changes associated with such properties (Walker et al. 2004; Scheffer et al. 2015). For this purpose, we have defined and illustrated-for the first time to our knowledge-a powerful model class to formalize ecosystem behaviors. No restriction on types of ecological components (biotic, abiotic or anthropic) and interactions (e.g. ecological or socio-economical) are imposed by such integrated models. Petri nets, already widely used in biology and in social sciences (Murata 1989; Blätke, Heiner \& Marwan 2011; Koch, Reisig \& Schreiber 2011) along with other qualitative models (Kauffman 1969; Thomas \& Kaufman 2001), have demonstrated here their ability to model a theoretical complex ecosystem.

The Petri net model developed here rigorously and successfully computed every possible state and trajectory encountered by the termite ecosystem (Fig. 4a). The model also automatically computed 
qualitatively stable (resilient, SCC) and unstable (tipping point) zones (Fig. 4b). Although qualitative, such models can compute many quantitative metrics from the large state space provided.

\section{Understanding the termite ecosystem}

The termite colony was represented as a relational network between biotic and abiotic components that was allowed to initiate, grow and die, depending on ecological rules and disturbances applied to the network (Fig. 4). We stress the conceptual difference between system functioning, focusing on the (often short term) abundance and flux variations between system components, whilst keeping the system structure unchanged, and system development, focusing on the (often long term, topological) system structure changes (Odum \& Odum 1971; Gaucherel et al. 2017). While this qualitative model allows the modeling of a large and complex interaction network, it identifies only possible fates, without their occurrence probabilities. As a perspective, a quantitative Petri net model would also provide the confidence interval for frequent (highly probable) and unrealistic (highly improbable) trajectories.

As soon as the ecosystem model is built and its simplifications assumed, the state space of the modeled ecosystem is automatically computed and reveals insights into the ecosystem dynamics (called the development, Fig. 4). Starting with the system growth from an initial state composed only of reproductives (and the permanent soil and atmosphere environment), two kinds of colony collapse can occur. Collapse of the termite colony, i.e. states in which the system is paralyzed, are always reached after reproductive death, due to the lack of breathing air in the nest (rule 13) or an attack by ant competitors (rule 12) (Turner 2009). Reproductives are the sole component in the model that are able to produce workers and egg chambers (rules 4 and 2). Hence, our model allows for the rigorous detection of decisive rules, i.e. tipping points with irreversible effects, that inevitably lead to system collapse (Costa \& Fitzgerald 2005; Turner 2009). Conversely, if reproductives remain alive, the system may remain indefinitely in similar states by first producing workers and 
termitomyces (mushrooms), and then by traveling through many trajectories of creation/destruction cycles. The "resilient" states (SCCS B and B') act as structural basins of attraction and remain unchanged as long as no ant competitors arrive in the system. Such non-trivial global features (see Appendix 3 for other added values) would not have been detected without the use of this model exploring exhaustively the system behavior.

These results confirm the central roles of the reproductives and air quality of the nest, as well as the minor roles of workers, soldiers and other components that the termite system can rapidly regenerate, as found in real colonies (Costa \& Fitzgerald 2005; Turner 2009). The result also highlights the importance of modeling assumptions. For example, adding a new rule allowing the workers to regenerate reproductives would have drastically changed the ecosystem fates, while many other rules would not have changed the state space at all. Adding such a decisive rule mimics ecological engineering practices designed to restore a disturbed ecosystem (DeAngelis et al. 1998; Pfadenhauer 2001). From another perspective, our discrete model could also be used to qualitatively study the system's sensitivity to additional rules (ecological processes) and the way such rules may degrade or improve the ecosystem's resilience (rules 2 and 4, or 7, Fig. 5). The sensitivity of discrete models to changes in the rules driving the behavior has also been studied in the context of Boolean networks applied to the modelling of biological systems (Saadatpour et al. 2011; Gaucherel et al. 2017).

\section{Discrete and qualitative ecosystem models}

The main originality of our work not only lies in the use of discrete models (e.g. Petri nets) in ecology, as some other studies started to explore the promising avenue of discrete models too (Ewing et al. 2002; Gaucherel et al. 2012; Baldan et al. 2015; Gaucherel et al. 2017). Rather, it concerns the discrete and qualitative conception of the integrated ecosystem forming, we think, the backbone of any ecosystem. The discrete engine behind our model then allows an exhaustive and rigorous exploration of the ecosystem behaviour. This observation is striking considering that almost all 
ecosystem or species community models focus on more continuous and gradual changes (Thébault \& Fontaine 2010; Kéfi et al. 2016).

The formal modeling of dynamic ecological systems has traditionally been carried out using differential equations (Thébault \& Fontaine 2010; Kéfi et al. 2016). However, discrete modelling developed in computer sciences has proven to be successful, in particular in systems biology (Lindenmayer 1978; Campbell et al. 2011; Gaucherel et al. 2012). The two approaches can be considered as complementary: while traditional differential equations usually provide quantitative mean field behavior for the system, discrete modelling enables a qualitative analysis of every behavior. This is notably done through the inventory of the system states, of some system characteristic events, through the decomposition of the system trajectories, of causality, and of independence (Pommereau 2010; Koch, Reisig \& Schreiber 2011). Discrete models often capture the trajectories of the dynamic system more accurately (Cordier, Largouët \& Zhao 2014; Gaucherel et al. 2017). For example, in the termite colony we modeled, differential equations would have certainly identified the pivotal role of rules 12 and 13 in the system collapse (Fig. $4 a-C$ ), but it would have been difficult to confirm that these rules are the only ones that are dangerous for the colony. For the same reason, differential equations would not have easily identified and characterized the termite colonyspecific scenarios on both sides of the tipping point. While some property identifications here could have been perceived a posteriori as trivial, Petri nets show the same power for identifying any (potentially complex) basins and tipping points.

Our formal model defined two semantics that we showed were equivalent. The first semantics is called operational, because it defines states and provides the operations to execute one transition (rule) to reach another state. The second semantics is called denotational because it transforms states and rules into new objects, i.e., Petri nets that are associated with their own semantics. Operational semantics is useful to describe ecological aims with formal modeling as directly as possible: it rigorously grasps the notions needed to understand the ecosystem at the adequate level 
of abstraction (Koch, Reisig \& Schreiber 2011). However, semantics is a mathematical definition only, and no software tool exists to handle it. These tools may be developed, but it is more straightforward to define an equivalent denotational semantics and the tool required for the translation only.

To define the denotational semantics of this study, we chose a Petri net translation well adapted to network handling, although other formalisms exist (Lindenmayer 1978; Thomas \& Kaufman 2001; Gaucherel et al. 2012; Giavitto, Klaudel \& Pommereau 2012). Petri nets are widely acknowledged as a useful modelling formalism for building discrete models of biological systems. Among their benefits, the following are often emphasized (Machado et al. 2009): simplicity, as Petri nets are easy to understand and adopt for biological situations, while still allowing a large flexibility (called expressivity); graphical representation that both conveys intuition and allow topological analysis; modularity that allows the building of large models by assembling smaller blocks and the potential to be extended in many different ways (see below).

\section{On the benefits of Petri nets}

We note three key benefits of using Petri nets: the adequacy to express the denotational semantics of our nodes/rules-based formalism, the wide range of analyzing techniques and available tools, and the flexibility that opens the way to future extensions. For example, it is straightforward to add features like priorities, time, stochasticity and quantitative data (David \& Alla 2010). Any Petri net may become quantitative by adding a number of tokens in various places (multivalued nets, Pommereau 2010; Reisig 2013). This flexibility allows us to foresee in ecology many possible extensions of the current setting. Yet, the 'reachability graph' of Petri nets suffers from the wellknown 'state explosion' problem, that is, the reachability graph may be exponentially larger than the corresponding Petri net. This observation limits the size of the models providing an explicit computation of their reachability graphs, although we already manage large graphs composed of more than four million states. However, symbolic techniques exist, such as decision diagrams (Hamez, Thierry-Mieg \& Kordon 2008), to alleviate the explosion of the state space. 
When considering extensions to Petri nets, a wide range of methods and software tools are available to develop and analyze them (Haustermann et al. 2017). Here, we used a custom marking graph analysis, with the aggregation of strongly connected components and the basins (Fig. 4). We used the SNAKES toolkit (Pommereau 2015) to implement the denotational semantics, the TINA toolkit to compute the marking graph (Berthomieu 2017), and custom tools to perform the analyzing steps. In the future, we plan to extend the structural analysis available with Petri nets and in particular to exploit its transitions- and places-invariants, or traps.

To adapt Petri nets for an ecological system, we first designed a modelling language (reaction rules) intended to be directly used by ecologists and to explicitly define the concepts dealt with, e.g., ecological components, and elementary processes. This formalism has to be self-contained and to include operational semantics, including diagnostics, to allow the modeler a high-level of abstraction. In addition, we need a denotational semantics to access existing analyses and software tools. Petri nets are suitable for translating the high-level formalism, as nodes and rules naturally map onto places and transitions, respectively. Other methods such as automata or process algebra are also well suited to define parallel processes, but communication is usually handled through transition synchronization, whereas ecologists need resource sharing with concurrent accesses. Boolean networks are well suited for modelling ecological nodes (Gaucherel et al. 2017), but they handle deterministic processes only and are not as open to extensions as in Petri nets. Petri nets are probably the most versatile discrete modeling framework in life sciences (Machado et al. 2009), which explains their recent popularity growth in systems biology.

\section{Ecosystem resilience and tipping points}

The Petri net model is powerful in describing ecosystem development, and other ecosystem-related concepts. SCCs and associated basins play the role of relatively stable zones (Walker et al. 2004; Scheffer et al. 2015; Karssenberg, Bierkens \& Rietkerk 2017), as the termite ecosystem may run indefinitely into such structures. Such resilient states of the ecosystem, automatically identified by 
the Petri net analysis, are reminiscent of phenotypes of a living organism in biology (Blätke, Heiner \& Marwan 2011; Koch, Reisig \& Schreiber 2011). Phenotypes can be likened to basins keeping the system in a specific set of states although, in our model, such zones locally "attract" the system only when it enters these SCCs. It is possible to quantify the width of the basin by the number of states it contains (or by any other related variable) (e.g. 20 states in SCC B', Fig. 4 and Fig. S3). It is also possible to quantify the precariousness of the system at any state by the distance to exit the SCC, while the maximal distance to exit may naturally quantify the maximal system resilience in this basin (e.g., a depth of 3 in SCC B', Fig. S3). In addition, any shift between distinct SCCs or between any SCC and a basin containing a deadlock corresponds precisely to tipping points (Hirota et al. 2011; van Nes et al. 2014). The state-space provides a rigorous way to exhaustively identify these ecological features and other, e.g. specific trajectories, for any ecosystem, theoretically and as long as the system has been accurately understood and modeled.

So far, we have justified the qualitative conception of ecosystems by long term dynamics under study. However, our model is not dedicated to any spatial or temporal scale or to closed ecosystems, as matter and energy inputs(e.g. due to global changes or to evolution, Weber et al. 2017) may be integrated as additional nodes. The qualitative assumption rather assumes that the ecosystem graph forms the backbone of the system and provides deep insight into its overall behaviour (called the ecosystem development, Gaucherel et al. 2017). In the future, we aim to apply Petri nets to more complex spatial and temporal scales, ranging from a small pond over days to a continent over million years, with coherent management of each scale. In addition to methodological advantages previously mentioned, the advantages we see for all these ecological applications of discrete and qualitative models are the abilities: i) to integrate components of distinct natures, ii) to easily handle changing topologies of the ecological interactions involved, iii) to formalize the model and rigorously demonstrate its outputs, and iv) to capture the causality of the system dynamics (in the state space). 
534 In conclusion, we suggest that Petri nets provide a new class of models for analyzing ecosystem

535 behaviors and formalizing ecosystem development over the long term. The development concept is 536 fully coherent with drastic regime shifts and with resilience in ecosystem dynamics, and Petri nets 537 are a powerful and rigorous class of discrete models able to identify, handle and quantify ecosystem538 related concepts. In our conceptual view, any ecosystem may be modelled in an integrated way and 539 such a model provides the exhaustive trajectories based on the model assumptions. Using Petri nets 540 supposes that every ecosystem can be represented on the basis of its relational graph (interaction 541 network) connecting every relevant component of the ecosystem through any relevant process. The 542 ecosystem graph is then rigorously handled with a discrete model exhaustively modifying graph 543 topology, which is the real added value of our model. To our knowledge, this approach has not yet 544 been used in an example of an ecological situation. The existence of a wide range of disturbed 545 ecological networks opens an avenue to this new way of identifying resilience and tipping points over 546 the long term. 


\section{References}

Baldan, P., Bocci, M., Brigolin, D., Cocco, N. \& Simeoni, M. (2015) Petri nets for modelling and analysing trophic networks. BioPPN 2015, a satellite event of PETRI NETS 2015 (eds M. Heiner \& A.K. Wagler). CEUR Workshop Proceedings.

Berthomieu, B. (2017) The TINA toolkit. http://projects.laas.fr/tina//home.php.

Best, E., Devillers, R. \& Koutny, M. (2001) Petri Net Algebra. Springer, Berlin.

Blätke, M.A., Heiner, M. \& Marwan, W. (2011) Tutorial. Petri Nets in Systems Biology. Otto-vonGuericke University Magdeburg, Germany.

Campbell, C., Yang, S., Albert, R. \& Sheab, K. (2011) A network model for plant-pollinator community assembly. proceedings of the National Academy of Sciences (PNAS), 108, 197-202.

Carpenter, S.R. (2003) Regime shifts in lake ecosystems. Ecology Institute, Oldendorf/Luhe, Germany.

Cordier, M.-O., Largouët, C. \& Zhao, Y. (2014) Model-Checking an Ecosystem Model fo Decision-Aid. IEEE 26th International Conference on Tools with Articficial Intelligence, pp. 539-543. IEEE Computer Society.

Costa, J.T. \& Fitzgerald, T.D. (2005) Social terminology revisited: Where are we ten years later? Ann. Zool. Fennici, 42, 559-564.

David, R. \& Alla, H. (2010) Discrete, Continuous, and Hybrid Petri Nets. Springer, Berlin, Heildelberg, Germany.

DeAngelis, D.L. (1980) Energy-flow, nutrient cycling, and ecosystem resilience. Ecology, 61, 764-771.

DeAngelis, D.L., Gross, L.J., Huston, M.A., Wolff, W.F., Fleming, D.M., Comiskey, E.J. \& Sylvester, S.M. (1998) Landscape modeling for everglades ecosystem restoration. Ecosystems, 1, 64-75.

Ewing, B., Yandell, B., Barbieri, J., Luck, R. \& Forster, L. (2002) Event-driven competing risks. Ecological Modelling, 158, 35-50.

Fowler, H.G., Pereira da-Silva, V., Forti, L.C. \& Saes, N.B. (1986) Population dynamics of leaf cutting ants: A brief review. Fire ants and leaf-cutting ants, pp. 123-145. Westview Press, Colorado, Boulder. 
Gaucherel, C. (2010) Self-organization of patchy landscapes: Hidden optimization of ecological processes. Journal of Ecosystem \& Ecography, 1.

Gaucherel, C., Boudon, F., Houet, T., Castets, M. \& Godin, C. (2012) Understanding Patchy Landscape Dynamics: Towards a Landscape Language. PLOS ONE, 7, e46064.

Gaucherel, C., Théro, H., Puiseux, A. \& Bonhomme, V. (2017) Understand ecosystem regime shifts by modelling ecosystem development using Boolean networks. Ecological Complexity, 31, 104114.

Giavitto, J.-L., Klaudel, H. \& Pommereau, F. (2012) Integrated regulatory networks (IRNs): Spatially organized biochemical modules. Theoretical Computer Science, 431 219-234.

Hamez, A., Thierry-Mieg, Y. \& Kordon, F. (2008) Building efficient Model-Checkers using hierarchical Set Decision Diagrams and Automatic Saturation. Fundamenta Informatica Petri Nets (IOS Press), 1-25.

Haustermann, M., Wagner, T., Moldt, D., Heitmann, F., Rölke, H., Mortensen, K.H., Kummer, O. \& Christensen, S. (2017) Petri Nets Tool Database. Petri Nets World.

Hély, C., Braconnot, P., Watrin, J. \& Zheng, W. (2009) Climate and vegetation : Simulating the African Humid Period. Comptes Rendus Géoscience, 341, 671-688.

Hély, C., Shuggart, H.H., Swap, B. \& Gaucherel, C. (2019) The Drape Concept to Understand Ecosystem Dynamics and its Tipping Points. Submitted.

Hirota, M., Holmgren, M., Van Nes, E.H. \& Scheffer, M. (2011) Global Resilience of Tropical Forest and Savanna to Critical Transitions. Science, 334, 232-235.

Holling, C.S. (1973) Resilience and stability of ecological systems. Annu. Rev. Ecol. Syst., 4, 1-23.

Karssenberg, D., Bierkens, M.F.P. \& Rietkerk, M. (2017) Catastrophic Shifts in Semiarid VegetationSoil Systems May Unfold Rapidly or Slowly. American Naturalist, 190, E145-E155.

Kauffman, S.A. (1969) Metabolic stability and epigenesis in randomly constructed genetic nets. Journal of theoretical biology, 22, 437-467. 
Kéfi, S., Miele, V., Wieters, E., Navarrete, S. \& Berlow, E. (2016) How Structured Is the Entangled Bank? The Surprisingly Simple Organization of Multiplex Ecological Networks Leads to Increased Persistence and Resilience. PLoS Biology, 14, e1002527.

Koch, I., Reisig, W. \& Schreiber, F. (2011) Modeling in systems biology. The Petri net approach. Springer-Verlag, London, UK.

Lenton, T.M., Livina, V.N., Dakos, V. \& Scheffer, M. (2012) Climate bifurcation during the last deglaciation? Climate of the Past, 8, 1127-1139.

Lindenmayer, A. (1978) Algorithms for plant morphogenesis. Theoretical plant morphology (ed. R. Sattler), pp. 37-81. Leiden University Press, The Hague.

Machado, D., Costa, R.S., Rocha, M., Rocha, I., Tidor, B. \& Ferreira, E.C. (2009) A Critical Review on Modelling Formalisms and Simulation Tools in Computational Biosystems. International Work-Conference on Artificial Neural Networks (ed. A.I. IWANN : Distributed Computing, Bioinformatics, Soft Computing, and Ambient Assisted Living), pp. 1063-1070. Part of the Lecture Notes in Computer Science book series (LNCS).

Murata, T. (1989) Petri nets: Properties, analysis and applications. Proceedings IEEE, 77, 541-580.

Odum, E.P. \& Odum, H.T. (1971) Fundamentals of ecology, 3rd edn. Saunders, Philadelphia, US.

Pfadenhauer, J. (2001) Some remarks on the socio-cultural background of restoration ecology. Restoration Ecology, 9, 220-229.

Pommereau, F. (2010) Algebras of coloured Petri nets. Lambert Academic Publishing (LAP).

Pommereau, F. (2015) SNAKES. A flexible high-level Petri nets library. Proceedings of PETRI NETS'15. LNCS 9115. Springer

Reisig, W. (2013) Understanding Petri Nets. Springer Berlin Heidelberg, Berlin, Heidelberg.

Saadatpour, A., Wang, R.-S., Liao, A., Liu, X., Loughran, T., Albert, I. \& Albert, R. (2011) Dynamical and Structural Analysis of a T Cell Survival Network Identifies Novel Candidate Therapeutic Targets for Large Granular Lymphocyte Leukemia. PLoS Comput Biol, 7, e1002267.

Scheffer, M. (2009) Critical Transitions in Nature and Society. Princeton Univ. Press, Princeton, NJ. 
Scheffer, M., Carpenter, S., Foley, J.A., Folke, C. \& Walker, B. (2001) Catastrophic shifts in ecosystems. Nature, 413, 591-596.

Scheffer, M., Carpenter, S.R., Dakos, V. \& van Nes, E.H. (2015) Generic Indicators of Ecological Resilience: Inferring the Chance of a Critical Transition. The Annual Review of Ecology, Evolution, and Systematics, 46, 145-167.

Scheffer, M., Hosper, S.H., Meijer, M.L., Moss, B. \& Jeppesen, E. (1993) Alternative equilibria in shallow lakes. TRENDS in Ecology and Evolution, 8, 275-279.

Thébault, E. \& Fontaine, C. (2010) Stability of Ecological Communities and the Architecture of Mutualistic and Trophic Networks. Science, 329, $853-856$.

Thom, R. (1975) Structural stability and morphogenesis. Benjamin, Reading, MA.

Thomas, R. \& Kaufman, M. (2001) Multistationarity, the basis of cell differentiation and memory. II. Logical analysis of regulatory networks in terms of feedback circuits. Chaos, 11, 180-195.

Turner, J.S. (2009) The extended organism: the physiology of animal-built structures. Harvard University Press.

van Nes, E.H., Hirota, M., Holmgren, M. \& Scheffer, M. (2014) Tipping points in tropical tree cover: linking theory to data. Global Change Biology, 20, 1016-1021.

Van Nes, E.H. \& Scheffer, M. (2007) Slow Recovery from Perturbations as a Generic Indicator of a Nearby Catastrophic Shift. American Naturalist, 169, 738-747.

Walker, B., Holling, C.S., Carpenter, S.R. \& Kinzig, A. (2004) Resilience, adaptability and transformability in social-ecological systems. Ecology and Society, 9, 5.

Weber, M.G., Wagner, C.E., Best, R.J., Harmon, L.J. \& B. Matthews (2017) Evolution in a Community Context: On Integrating Ecological Interactions and Macroevolution. Trends in Ecology \& Evolution, 32, XXX. 
651

652 Author contributions: C.G. and F.P. conceived the ideas and the model, analysed its outputs and led 653 the writing together.

654

655

656

657

658

659

660

661

662

663

664

665

666

Appendix 3: State-space refined analysis

\section{Acknowledgments}

We thank Cinzia Di Giusto for discussions on ecosystem definition and Serguei Verlan for discrete model specificities.

\section{Data Accessibility}

Readers interested may find the PNML file at this URL https://zenodo.org/record/3241370 (DOI : 10.5281/zenodo.3241370)

\section{SUPPLEMENTARY MATERIAL - APPENDICES}

Appendix 1: The functioning of termite and ant colonies

Appendix 2: Proof of theorem 1

667 
672

\begin{tabular}{|c|c|c|c|c|}
\hline Name & initially & family & description & comment \\
\hline$R p$ & present & inhabitants & reproductives & $\begin{array}{l}\text { the queen, the king, the eggs and the } \\
\text { nymphs }\end{array}$ \\
\hline$W k$ & absent & inhabitants & workers & $\begin{array}{l}\text { all termites able to work: the larvae, } \\
\text { workers, pseudo-workers }\end{array}$ \\
\hline$S d$ & absent & inhabitants & soldiers & the termite soldiers \\
\hline Te & absent & inhabitants & termitomyces & the fungus cultivated by the termites \\
\hline$E c$ & absent & structures & egg chambers & all egg chambers plus the royal chamber \\
\hline$F g$ & absent & structures & fungal gardens & all the gardens in which the fungus is grown \\
\hline$M d$ & absent & structures & mound & the upper structure of the colony \\
\hline$W d$ & absent & resources & wood & the wood stored inside the colony \\
\hline$A i$ & absent & resources & air of the nest & the air inside the colony \\
\hline$S l$ & present & environment & soil & the soil around the termite nest \\
\hline$A t$ & present & environment & atmosphere & the air around the termite nest \\
\hline Ac & present & competitors & ant competitors & $\begin{array}{l}\text { all the ant species in competition with the } \\
\text { termites }\end{array}$ \\
\hline
\end{tabular}

673 
675 Table 2. List of the rules modeling the termite ecosystem functioning and development. The order of 676 priority (sequence), the conditions of application, the products (consequences), and a detailed explanation are given for each rule. The rule arrows indicate the transformation (rewriting) of the network at next step.

679

\begin{tabular}{|c|c|}
\hline Rule & comment \\
\hline 1: $W k^{+}, T e^{+} \rightarrow W d^{-}, A i^{-}$ & $\begin{array}{l}\text { the workers and the fungi are consuming wood } \\
\text { and air }\end{array}$ \\
\hline $2: F g^{-} \rightarrow T e^{-}$ & $\begin{array}{l}\text { the fungi need the fungal gardens in order to } \\
\text { survive }\end{array}$ \\
\hline 3: $W k^{+}, S l^{+} \rightarrow W d^{+}, T e^{+}, F g^{+}, E c^{+}, M d^{+}$ & $\begin{array}{l}\text { the workers are foraging in the soil for wood and } \\
\text { fungus; from the soil, the workers are building } \\
\text { the fungal gardens, the egg chambers, and the } \\
\text { mount }\end{array}$ \\
\hline $4: W d^{-} \rightarrow W k^{-}, T e^{-}$ & $\begin{array}{l}\text { the workers and the fungus need to eat wood to } \\
\text { survive }\end{array}$ \\
\hline $5: R p^{+}, S l^{+} \rightarrow E c^{+}$ & $\begin{array}{l}\text { for the soil, the queen and the king can also build } \\
\text { egg rooms }\end{array}$ \\
\hline 6: $R p^{+}, E c^{+} \rightarrow W k^{+}$ & $\begin{array}{l}\text { in the egg chambers, the queen and the king are } \\
\text { producing eggs that are becoming workers }\end{array}$ \\
\hline $7: W k^{+}, W d^{+} \rightarrow S d^{+}, R p^{+}$ & $\begin{array}{l}\text { eating some wood, the larvae are } \\
\text { metamorphosing into soldiers and/or nymphaea }\end{array}$ \\
\hline 8: $M d^{+}, A t^{+} \rightarrow A i^{+}$ & $\begin{array}{l}\text { the air of the nest is being refreshed by passing } \\
\text { through the mound and exchanging with the } \\
\text { atmosphere }\end{array}$ \\
\hline
\end{tabular}




\begin{tabular}{|c|c|}
\hline 9: $W k^{-} \rightarrow F g^{-}, S d^{-}$ & $\begin{array}{l}\text { the soldiers cannot survive without the workers } \\
\text { to feed them, and the fungal gardens need some } \\
\text { maintenance by the workers }\end{array}$ \\
\hline 10: $W k^{-}, R p^{-} \rightarrow E c^{-}$ & $\begin{array}{l}\text { the egg chambers need some maintenance by the } \\
\text { workers or the reproductives, otherwise they } \\
\text { collapse }\end{array}$ \\
\hline $11: S d^{+} \rightarrow A c^{-}$ & $\begin{array}{l}\text { the soldiers are killing ant competitors intruding } \\
\text { into the colony }\end{array}$ \\
\hline $12: A c^{+}, S d^{-} \rightarrow W k^{-}, R p^{-}$ & $\begin{array}{l}\text { without the soldiers, the ant competitors are } \\
\text { invading the colony and killing the workers and } \\
\text { the reproductives }\end{array}$ \\
\hline 13: $A i^{-} \rightarrow R p^{-}, W k^{-}, T e^{-}$ & $\begin{array}{l}\text { the reproductives, the workers and the fungus } \\
\text { need to breath the air of the nest to survive }\end{array}$ \\
\hline
\end{tabular}


682

683

684

685

686

687

688

689

690

691

692

693

694

695

696

697

698

699

700

701

702

703

704

705

\section{Figures}

Figure 1. Graphic of a termite colony (a) and its simplified interaction network (b). Termites modify their environment and build a mound with various chambers to host the colony (a). The original ecosystem graph is composed of 12 nodes (Table 1) with five colors representing their different natures (b, left). Their 15 respective interactions (processes, Table 2 ) are oriented (b, edges are directed from the thin to the thick end).

Figure 2. Illustration of a simplistic predator-prey system (a), with its qualitative dynamics (b), its associated Petri net (c) and marking graph (d). The system is made of two ecosystem components, the prey $(\mathrm{N})$ and predator $(\mathrm{P})$ populations, and two interactions connecting them (rules $\mathrm{R} 1$ and $\mathrm{R} 2$ ), as seen on the automaton (a). Starting with the presence of both populations, it is possible to list all system states encountered (b), and to connect them with the rules (absent components and inactivated rules are displayed in grey). The corresponding Petri net is made of four places ( $\mathrm{P}+, \mathrm{P}_{-}$, $\mathrm{N}+, \mathrm{N}-)$ and two transitions $\mathrm{R} 1$ and $\mathrm{R} 2$, where unlabeled arcs have weight 1 . The net is depicted in the initial state (c), and the successive states may be deduced from the token circulation seen in the dynamics (b). The marking graph of the Petri net $(d)$ is depicted with each state number $\left(S_{0}, S_{1}, S_{2}\right)$ referring to the dynamics described above (b). Notice that the (sole) specific state of the system $\left(\mathrm{S}_{3}\right)$ may not be reached from this initial condition and with these rules (d).

Figure 3. Focus on the rule 12 (Table 2) of the termite ecosystem modeled, based on the extended graph (a) and translation of the rule (b). Starting from the complete ecosystem (a, left), the rule 12: $A c^{+}, S d^{-} \rightarrow W k^{-}, R p^{-}$is drawn by the use of involved nodes only (a, right), where conditional nodes are shown in green and final nodes in gray. However, this diagram is not rigorous enough in 
that it does not make explicit how to proceed to apply the rule (see main text). The rule is therefore normalized into four distinct transitions (b). Each transition $t_{i}$ corresponds to a distinct normalized rule. In bold, the rule indeed fired.

Figure 4. The full (a) and reduced (b) marking graph of the Petri net provides the semantics of the termite model. The state-space is made of 109 states labeled with a pair $n / s$ where $n$ is an identifying number of the marking and $s$ is the number of the strongly connected states (SCC) and of the basin it belongs to. The initial state is displayed as a hexagon (A), deadlocks (states with no successors) are displayed as squares (five in total, among which two are in zones $D$ and $D^{\prime}$, and one is close to the initial state A), a tipping point is displayed as a red segment (C) and other states are displayed as circles. Each SCC or basin uses a distinct color (e.g. SCCs B and B' are drawn in orange and green). The edges are directed from the thin to the thick end and labeled with the number of the rule that was applied to perform the transition. The rule numbers are those of the ecological model (Table 2) and do not refer to the normalized rules (that are necessary technical steps, but do not make sense to the modeler). In the simplified and more abstract versions of the marking graph of a termite model (b), each SCC and basin has been reduced to a single node and redundant paths have been removed. Nodes representing aggregate SCCs or basins are noted ( $s$ ) (circles), more easily highlighting the sharp transitions between them. From this reduction of the marking graph, specific paths leading to the main ecosystem collapses can be more easily identified (squares). 
8

\section{Using discrete systems to exhaustively characterize the dynamics of an integrated ecosystem}

SUPPLEMENTARY MATERIAL - APPENDICES 
Macrotermitinae, also called fungus-growing termites, are an African and SE Asian subfamily of Termitidae (Engel \& Krishna 2004). The only known lineage of termites living in symbiosis with fungi, they are well known for the huge mounds they build. In the early stages of a (monogamous) colony, the (single) queen and king pair up during the nuptial flight (Shellman-Reeve 1990; Rosengaus \& Traniello 1991; Shellman-Reeve 1997), and then build a room in the soil. They lay eggs that develop into larvae. Larva development follows various pathways, the individuals becoming either workers or soldiers (the sterile line), or nymphaea that later develop into alates (the reproductive line) (Noirot 1955). Alates fly away during the next nuptial flight or, if needed, they can replace the royal pair or even transform into pseudo-workers (Noirot 1956). Termites experience hemimetabolous development, as immature stages have miniature adult morphology, and are therefore fit for labor.

Macrotermitinae live in a nest separated from their source of food (wood), so that workers have to forage outside the nest. During the first forage, the workers harvest the basidiospores of the Termitomyces species fungus (e.g. (Noirot 1955)) and grow them in gardens. Termites can digest the wood with the help of the preliminary fungus-performed lignin degradation process (Hyodo et al. 2000). The workers are also in charge of nest building, fungus care, and egg care. Soldiers protect the colonies and alates that are inactive. A mature termite nest contains a royal chamber, egg rooms, and fungus gardens below the surface and a mound above the surface, in order to regulate the composition and temperature of the air (Turner 2009). Many other species live inside their nest: fungus species of the Xylaria genus, the main parasites in the fungus garden, and other commensal species (Jaffe, Ramos \& Issa 1995), while ants, the primary predator of termites, can invade their colony (Leal \& Oliveira 1995).

To simulate the foundation of a termite colony (i.e. initial state), only the Soil, Atmosphere, Reproductives and Air nodes of the nest were set to On, the other nodes being absent (Off). Although Off nodes are present in the graph for modeling ease, they allow to mimic, by their very absence, a graph growth (i.e. development) coherent with other approaches in discrete modeling (Godin 2000; Giavitto \& Michel 2003; Sayama \& Laramee 2009). So, the Petri net developed here could later be improved into more complicated models. In order to capture the colony's dynamics, we then defined a finite set of rules establishing how node states changed over time. A transition (rule) is an oriented relationship between the states of nodes at time step $t$ and the states of the same or other nodes at time step $t+1$, according to certain conditions. In the case of the termite ecosystem and qualitative Petri net, transitions are simple, a state of "if then" (i.e. Boolean) rules (Thomas \& Kaufman 2001; Giavitto \& Michel 2003). All the (potentially applicable) rules were applied at each time step, checking

51 their associated conditions and whether they changed the state of the system or not (Tables

52 2), independently of the updated state of each node (i.e. each state was memorized and then taken as the initial state for all rule applications of the next step). 
Even when absent, the nodes pre-exist (i.e. the graph is fixed), thus highlighting the main difference with more complex graph-grammar models. For example, in the termite colony, the Wood node was Off at the beginning of the simulation, because this resource had not yet been introduced to the colony. This ecological process also meant that workers and wood nodes were connected by an oriented edge in the ecosystem's graph (Fig. 1a). For termite and ant ecosystems, extended graphs made up of 13 nodes were defined (Tables 1 and S1). Other graphs could have been chosen (and were tested in a sensitivity analysis), but led to comparable qualitative results. Ours were a compromise between over-detailed and over-simplified descriptions. Simplified five-node ecosystem graphs were built only to explain the influences of the rules (Fig. 1b).

\section{Appendix 2: Proof of theorem 1}

In this section, we intend to prove theorem 1 , which posits that the state space of $\left(\mathbb{E}, s_{0}, R\right)$ is isomorphic to the marking (or reachability) graph of $(S, T, W, M)$ (Fig. S1). This procedure is a common way of proceeding, although never done in the specific case of our two semantics (Best, Devillers \& Koutny 2001). First, we prove that the initial state and initial markings are equivalent. A state $s \subseteq \mathbb{E}$ corresponds exactly to the marking $\left\{e^{+} \mid e \in s\right\}+$ $\{\bar{e} \mid e \in \mathbb{E} \backslash s\}$, and conversely, the marking $M$ corresponds to the state $\left\{e \in \mathbb{E} \mid M\left(e^{+}\right)=1\right\}$. We note by $M(s)$ the marking that corresponds to state $s$. From item 1 in the definition of the Petri net semantics (see main text and Fig. S1), it follows that the initial marking is $M\left(s_{0}\right)$.

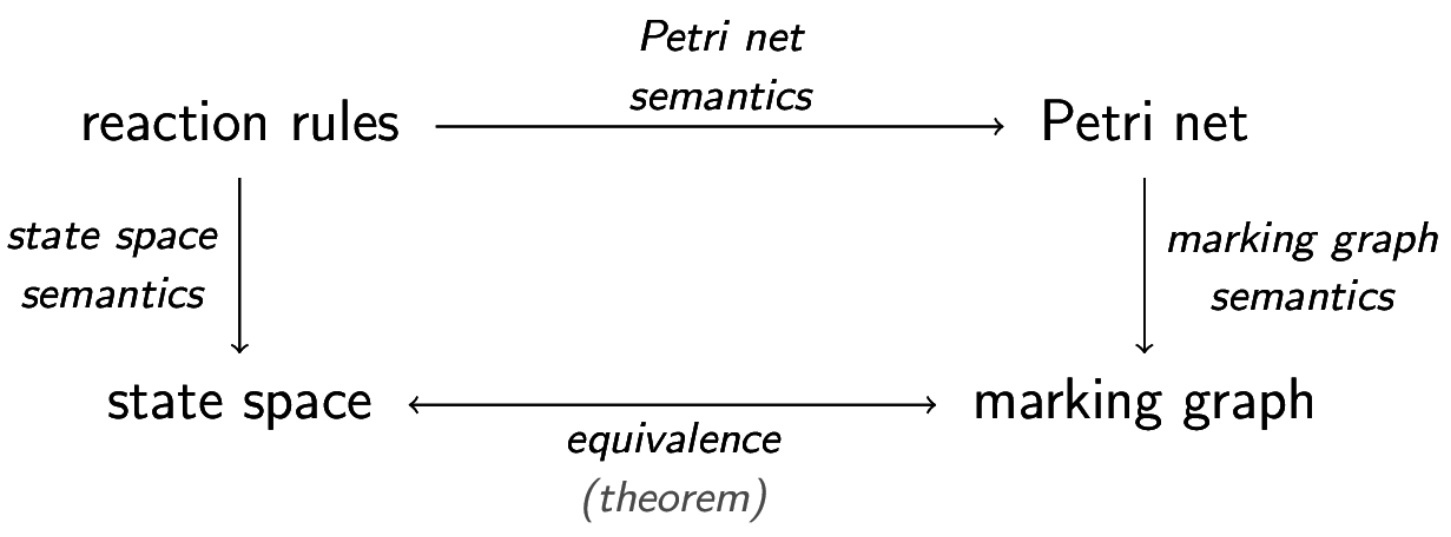

Figure S1. Diagram explaining the rigorous relationships between the various semantics involved in the study, and highlighting the equivalence between the marking graph and the computed state space.

We now prove that the state space and marking graph are constructed by two equivalent inductions. To do so, we must prove that $s \stackrel{r}{\rightarrow} s^{\prime}$ in the reaction rules correspond to the Petri net firing of $M(s)\left[t_{r^{\prime}}\right\rangle M\left(s^{\prime}\right)$ for some transition $t_{r^{\prime}}$ such that $r^{\prime}$ is a normalized rule obtained from $r$. There is an exact correspondence between normalized rules and transitions, so that we may equivalently use one or the other.

Let us first consider that we have $s \stackrel{\left(\alpha^{+}, \alpha^{-}, \omega^{+}, \omega^{-}\right)}{\longrightarrow} s^{\prime}$, and adopt the same notations $\alpha, \omega$, and $\chi$, as in the definition of normalized rules, and set $y \stackrel{\text { def }}{=} \chi \backslash x$. From the definition of firing 
rules, we have $\alpha^{+} \subseteq s$ and $\alpha^{-} \cap s=\emptyset$ and $s^{\prime} \stackrel{\text { def }}{=} s \backslash \omega^{-} \cup \omega^{+}$. From the definition of reaction rules, we also have $\alpha^{+} \cap \alpha^{-}=\omega^{+} \cap \omega^{-}=\emptyset$. All together, these constraints yield the Venn diagram (Fig. S2, diagram 1), while diagram 2 (Fig. S2) shows $s^{\prime}$. Applying the two steps of rule normalization, we see that $r$ is transformed into $r^{\prime} \stackrel{\text { def }}{=}\left(\alpha^{+} \cup x, \alpha^{-} \cup y, \omega^{+} \cup\right.$ $\left(\left(\alpha^{+} \cup x\right) \backslash \omega\right), \omega^{-} \cup\left(\left(\alpha^{-} \cup y\right) \backslash \omega\right)$. We, show $\chi$ in diagram 3 and take the partition $x \uplus$ $y$ such that $x$ corresponds to diagram 4 and $y$ corresponds to diagram 5 (Fig. S2). We can now verify that:

- $r^{\prime}$ is enabled because:

$\circ \alpha^{+} \cup x$, depicted as diagram 6 , is a subset of $s$,

$\circ \alpha^{-} \cup y$, depicted as diagram 7, does not intersect $s$;

- $s \stackrel{r^{\prime}}{\rightarrow} s^{\prime}$ because:

- let $p \stackrel{\text { def }}{=} \omega^{+} \cup\left(\left(\alpha^{+} \cup x\right) \backslash \omega\right)$ as depicted in diagram 8,

○ let $q \stackrel{\text { def }}{=} \omega^{-} \cup\left(\left(\alpha^{-} \cup y\right) \backslash \omega\right)$ as depicted in diagram 9,

$\circ$ we have $s^{\prime}=(s \backslash q) \cup p$.

Conversely, we can prove with exactly the same diagrams and a mirrored reasoning that if $s$ $\stackrel{r^{\prime}}{\rightarrow} s^{\prime}$, then we also have $s \stackrel{r}{\rightarrow} s^{\prime}$.
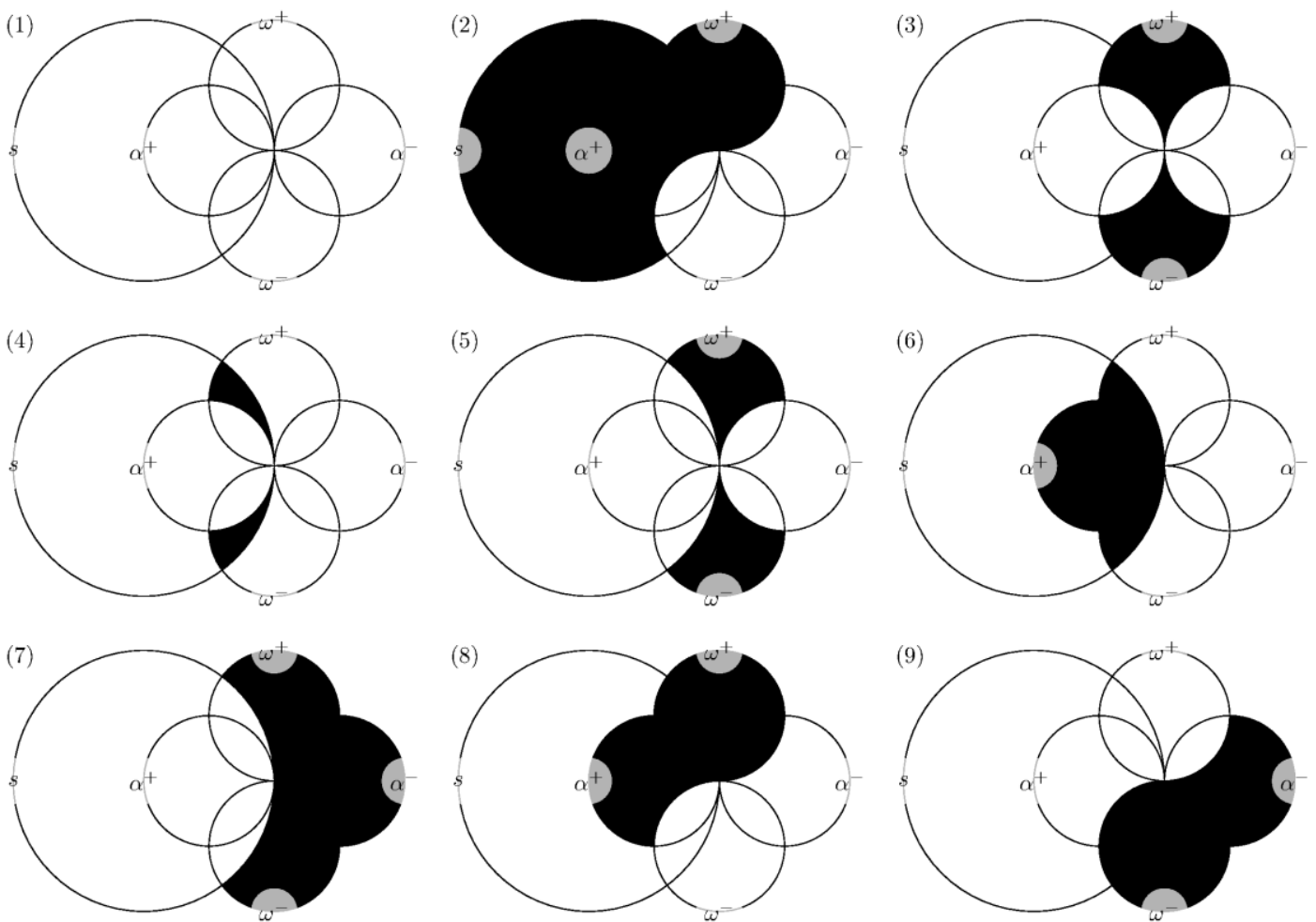

Figure S2. The distinct sets involved in the firing of a reaction rule $s \stackrel{\left(\alpha^{+}, \alpha^{-}, \omega^{+}, \omega^{-}\right)}{\longrightarrow} s^{\prime}$.

In addition, firing a rule independently to some others often leads to unrealistic paths (e.g. removing water without removing fishes in it). Therefore, we defined a new kind of rules called constraints, preventing the model from simulating such unrealistic paths. Constraints have a condition and a realization part, just as rules stricto sensu do, and model inevitable 
115 (mandatory) events given the system state. The sole difference between rules and 116 constraints is that constraints have priority on rules stricto sensu. In the prey-predator 117 system, the system state S1 is unrealistic; so, the rule R2 has to be transformed into a 118 constraint ( $\mathrm{C} 1: \mathrm{N}-, \mathrm{P}+\rightarrow \mathrm{P}-$ ). From a given state, the model first computes all trajectories 119 opened up by the defined constraints and then only, when all the system states obtained are 120 realistic (i.e. there is no longer any enabled constraint), the enabled rules are fired. In brief, 121 the discrete models proposed here is qualitative, mechanistic (processes are explicit), 122 deterministic (no stochasticity) and asynchronous (all rules are applied as soon as possible, no rule conflict as they systematically branch in the state space).

The termite ecosystem model combines one initial state, two strongly connected components (SCC) and four main deadlocks (Fig. 4). We may zoom into the SCC B' graph (Fig. S3a), as well as simplify it by merging each distance to exit (DTX) class into one new node to observe which rule allows moving from one class to another (Fig. S3b). The DTX class 0, only, is a SCC, while the other DTX classes are not strongly connected. For this reason, to merge the other classes may create paths that are not realizable in the modeled system. On this merged graph, hexagonal-shape nodes are those that contain at least one entrance of the SCC, while nodes are labeled with the DTX in parentheses to denote that this is a merged node.

Leaving DTX class 0 is always done through rule 8, while returning to class 0 is always done by decrementing DTX by one unit. Moreover, decreasing the DTX is always done using the same rules as shown by path $(3) \stackrel{6}{\rightarrow}(2) \stackrel{3}{\rightarrow}(1) \stackrel{1}{\rightarrow}(0)$. Finally, this merged graph is not complete: moving from a DTX class $x$ to a class $y>x$ is easier (i.e., there are more paths to do so) than moving in reverse $(x<y)$. So, as a second observation on the paths in our termite model, the system will eventually reach a deadlock if it executes a sequence finishing with $6,3,1$, and then 12 or 13 (in this order, but possibly interleaved with other rules). From SCC B, we can make the same observation, except that the system may execute rule 11 to reach SCC $B^{\prime}$. As a third observation, a random path within SCC B or $B^{\prime}$ is more likely to go through classes with higher DTX.

More details of the termite system behavior are available by removing all the edges between nodes of DTX class 0 , because class 0 is a SCC (we thus know that every node can be reached from another within this DTX class) (Fig. S3a). It appears that DTX class 2 is not connected and contains only transient states from which the system reaches DTX 1 or 3 . Moreover, the longest path within DTX class 1 is $97 \rightarrow 94 \rightarrow 25$, and within DTX class 3 it is $102 \rightarrow 84$ or $70 \rightarrow 84$. Therefore the system cannot stay for a long period without changing its DTX value. As a fourth observation, the system can stay within the same DTX class for short runs only: three successive states for DTX 1, one state for DTX 2, two states for DTX 3.

Although the system can reach any other class from DTX 0 , leaving DTX 0 is always done through rule 8 (Fig. S3b). As a fifth observation, the only way to increase DTX in SCC B', and thus to postpone the collapse, is to fire rule 8 . In addition, we observe that: i) a random path within any SCC ( $\mathrm{B}^{\prime}$ or $\left.\mathrm{B}\right)$ is more likely to move through classes with higher DTX; ii) the system can stay within the same DTX class for short runs only; and iii) the only way to 
increase DTX in SCC B', and thus to postpone the system collapse, is to fire rule 8. Finally, the model allows for accurate identification of the (simple, i.e. non-looping) trajectories of the system within each SCC.

Moreover, the largest SCC within this graph consists in all the states with non-zero DTX plus states 26 and 95 . Removing any of these two latter states also destroys the SCC B'. Hence, these states play central roles within SCC B' when we focus on paths trying to avoid DTX 0 . Finally, small SCCs composed of two states only may also be found: $\{70,85\},\{84-91\}$, and $\{102,107\}$. This means that avoiding DTX 0 is not possible on arbitrarily long trajectories without ultimately repeating these paths, yet with infinite runs alternating the two states of each couple. However, we may find an infinite number of paths without such oscillating paths that stay with DTX 0 for only one state (either 26 or 95).

(a)

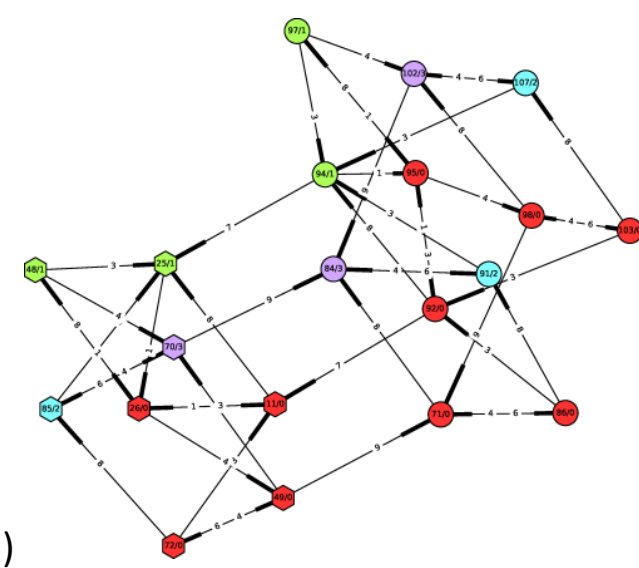

(b)

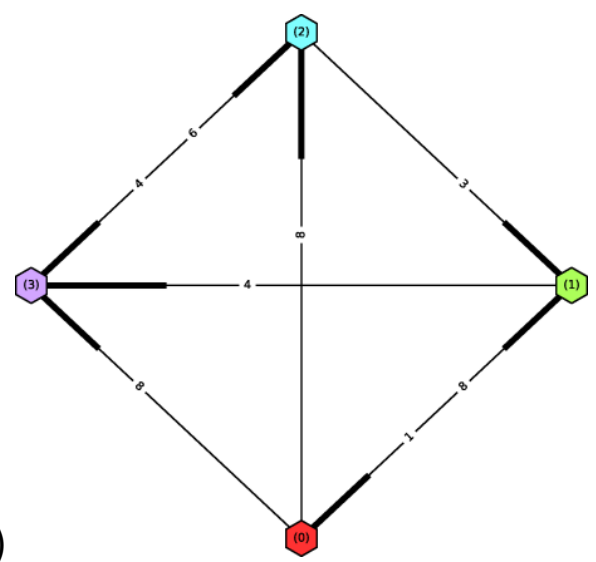

172

173

174

175

176

177

178

179

180

181

182

183

184

185

186

187

188

189

190

191

192

193

Figure S3. Zoom of the SCC B' (a) of the state-space (Fig. 4a), and a simplified version (b) in which each distance to exit (DTX) class has been merged into a single node. Here, nodes and interactions outside SCC B' have been hidden. Hexagonal-shaped nodes are those from which the system enters the SCC (8 possible states here). Nodes are labelled with pairs $n / d$ where $d$ and the color represent the distance to an exit node (DTX) of the focus state, that is, a state from which a rule may push the system outside the SCC (towards a deadlock, Fig. 4). In the simplified SCC (b), nodes are labeled with DTX and highlighted on both figures with a specific color (DTX=0 in red, 1-green, 2-cyan and 3-purple).

The simplified and trajectory-based version of the state space is informative too (Fig. 4b). From the SCC B, the system can reach SCC B' with rule 11 only (i.e. killing ant competitors), or the system can reach deadlock by first executing rule 12 (ants kill termites) or rule 13 (lack of air kills termites). From SCC B', rule 13 only remains possible because ant competitors are absent in the system. After one of these rules has pushed the system from any of its SCCs, several paths are available and correspond to the progressive depletion of resources in the system, which we may observe by plotting the paths (not depicted here). As a sixth and last observation for the termite system, the system dies if and only if it fires rule 12 or 13 (lack of reproductives or of fresh air, Table 2). Then, depending on the state from which this decisive rule is fired, the system executes four time steps (four rules) at most before collapsing. These results are contingent on the termite system modeled, but any (eco)system would benefit from this model ability to identify the decisive rules or sequences of rules and to quantify the dynamics associated with specific collapse or "alive" states (SCC). 


\section{References}

Best, E., Devillers, R. \& Koutny, M. (2001) Petri Net Algebra. Springer, Berlin. Engel, M.S. \& Krishna, K. (2004) Family-Group Names for Termites (Isoptera). American Museum novitates, 3432, 9.

Giavitto, J.L. \& Michel, O. (2003) Modeling the topological organization of cellular processes. Biosystems, 70, 149-163.

Godin, C. (2000) Representing and encoding plant architecture: A review. Annals of Forest Science, 57, 413-438.

Hyodo, F., Inoue, T., Azuma, J.I., Tayasu, I. \& Abe, T. (2000) Role of the mutualistic fungus in lignin degradation in the fungus-growing termite Macrotermes gilvus (Isoptera; Macrotermitinae). Soil Biology and Biochemistry, 32, 653658.

Jaffe, K., Ramos, C. \& Issa, S. (1995) Trophic interactions between ants and termites that share common nests. Annals of the Entomological Society of America, 88, 328-333.

Leal, I.R. \& Oliveira, P.S. (1995) Behavioral ecology of the neotropical termitehunting ant Pachycondyla (Termitopone) marginata: colony founding, groupraiding and migratory patterns. Behavioral Ecology and Sociobiology, 37, 373-383.

Noirot, C. (1955) Recherches sur le polymorphisme des termites supérieurs (Termitidae). Masson et Cie.

Noirot, C. (1956) Les sexués de remplacement chez les termites supérieurs (Termitidae). Insectes sociaux, 3, 145-158.

Rosengaus, R.B. \& Traniello, J.F. (1991) Biparental care in incipient colonies of the dampwood termite Zootermopsis angusticollis Hagen (Isoptera: Termopsidae). Journal of insect behavior, 4, 633-647.

Sayama, H. \& Laramee, C. (2009) Generative Network Automata: A Generalized Framework for Modeling Adaptive Network Dynamics Using Graph Rewritings. Adaptive Networks. Theory, models and applications (eds T. Gross \& H. Sayama), pp. 311-332. Springer, NECSI Cambridge/Massachusetts.

Shellman-Reeve, J.S. (1990) Dynamics of biparental care in the dampwood termite, Zootermopsis nevadensis (Hagen): response to nitrogen availability. Behavioral Ecology and Sociobiology, 26, 389-397.

Shellman-Reeve, J.S. (1997) Advantages of biparental care in the wood-dwelling termite, Zootermopsis nevadensis. Animal Behaviour, 54, 163-170.

Thomas, R. \& Kaufman, M. (2001) Multistationarity, the basis of cell differentiation and memory. II. Logical analysis of regulatory networks in terms of feedback circuits. Chaos, 11, 180-195.

Turner, J.S. (2009) The extended organism: the physiology of animal-built structures. Harvard University Press. 
a)
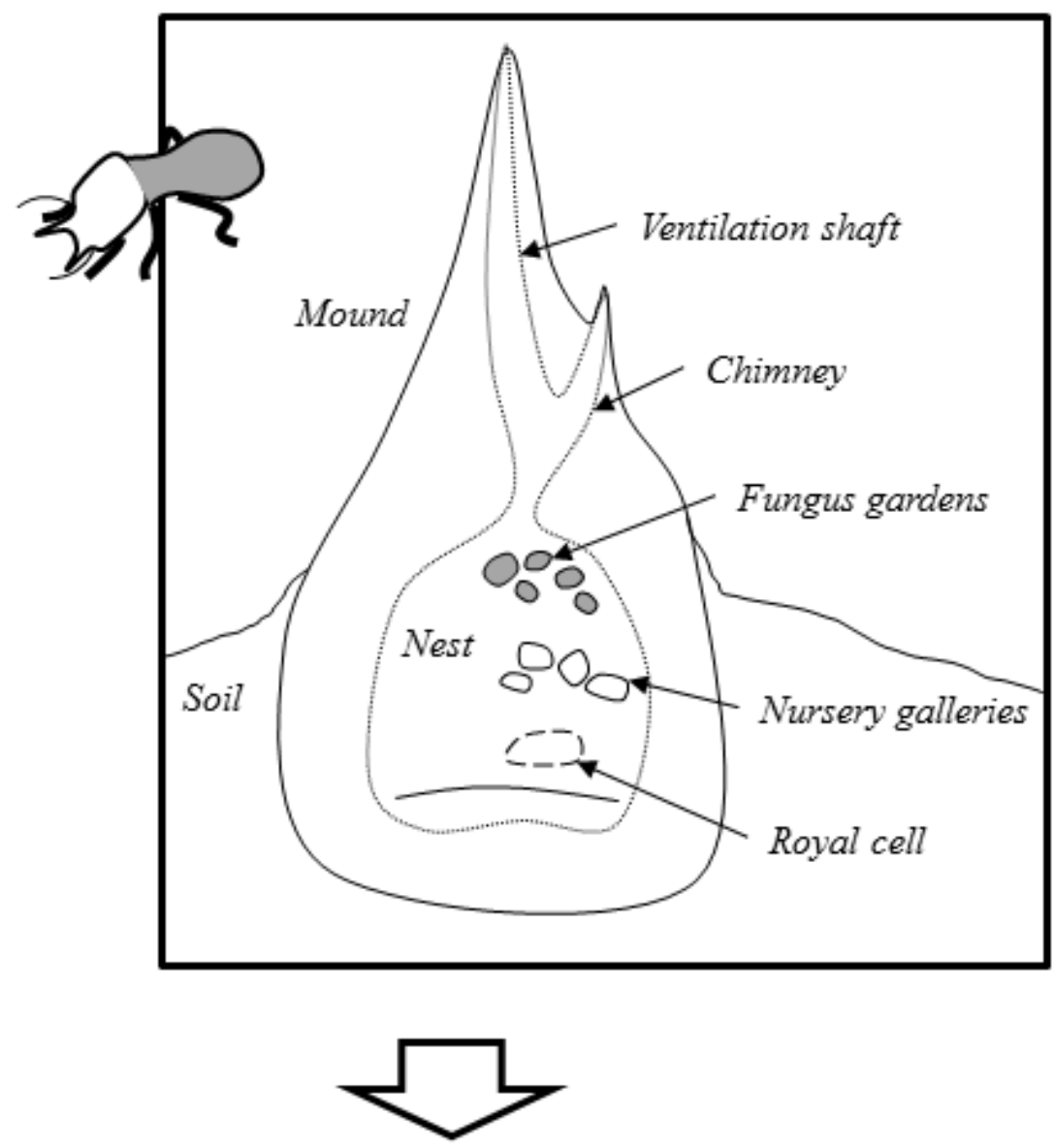

b)

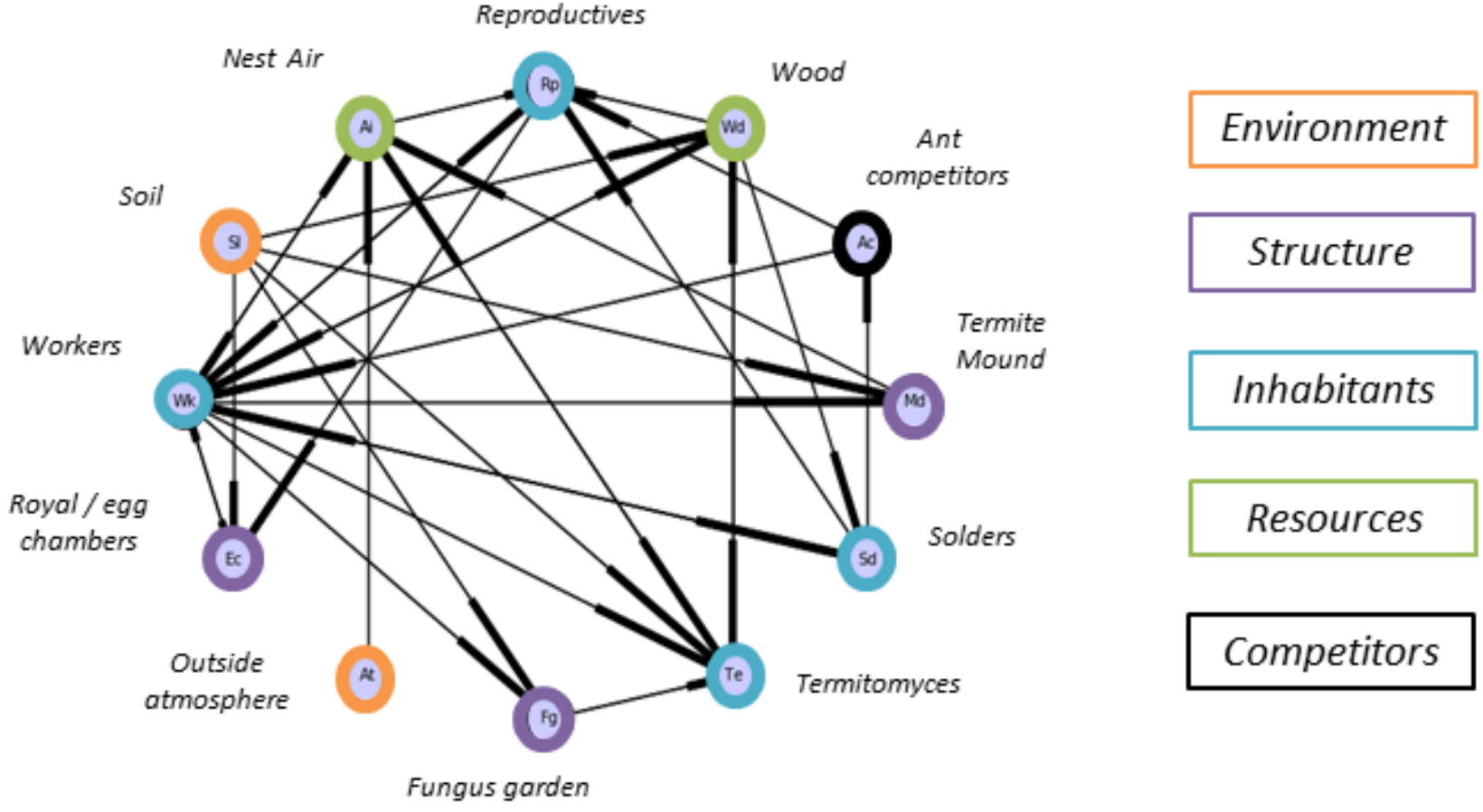

\section{FIGURE 1}


a)

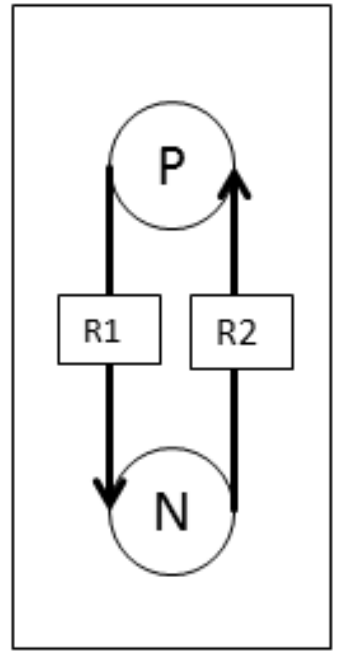

b)

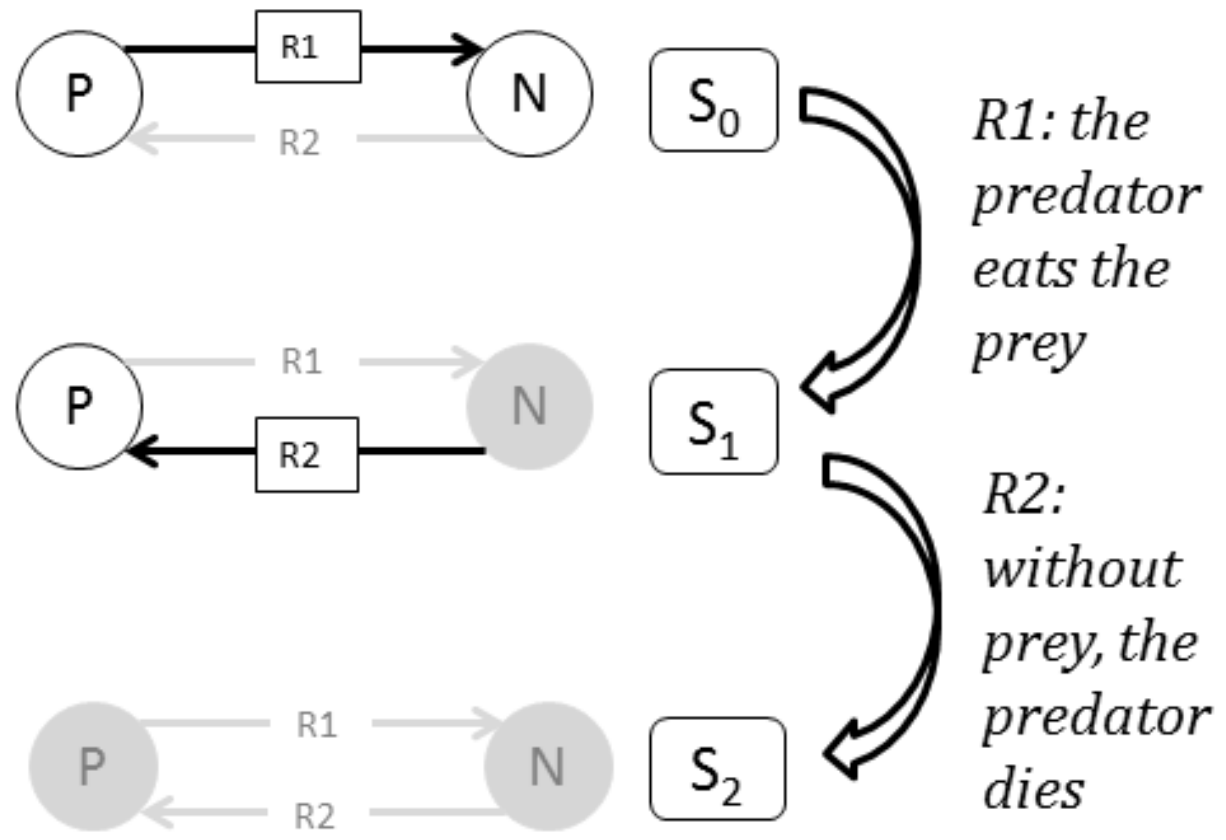

c)
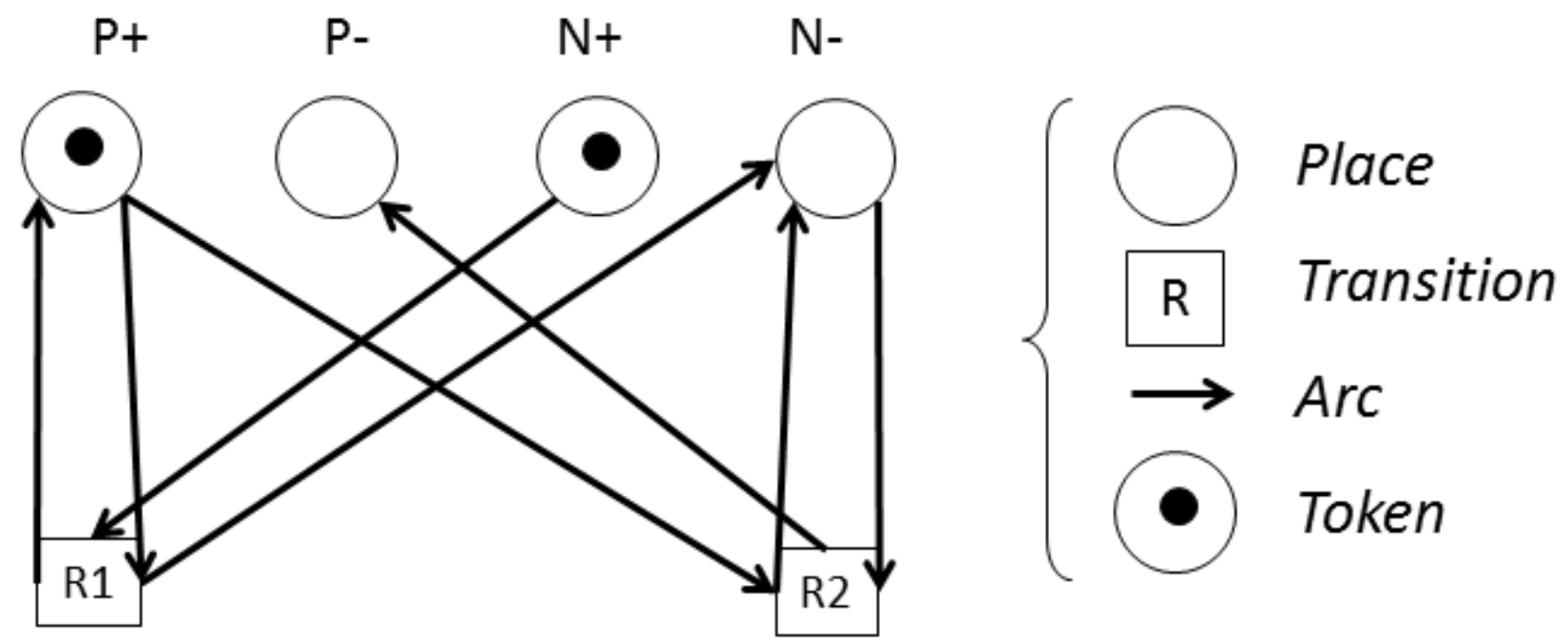

d)

FIGURE 2

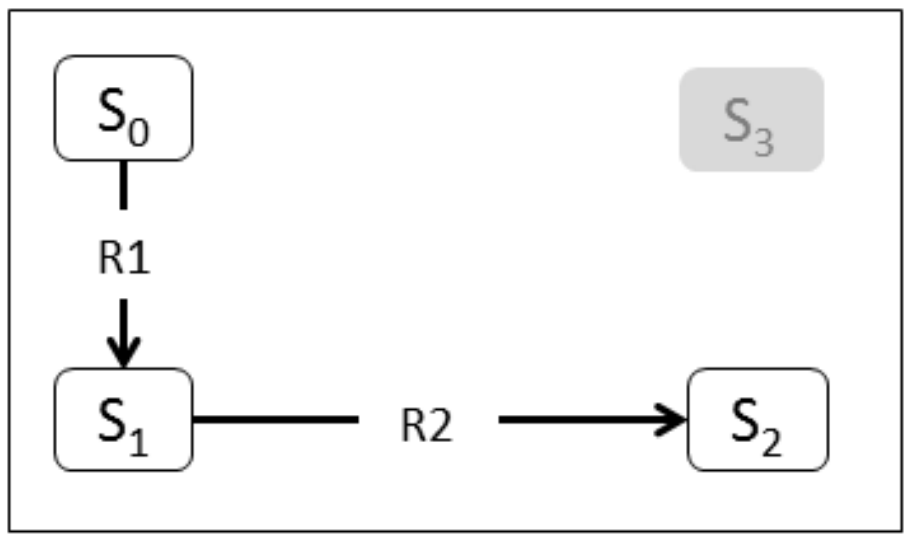


a)

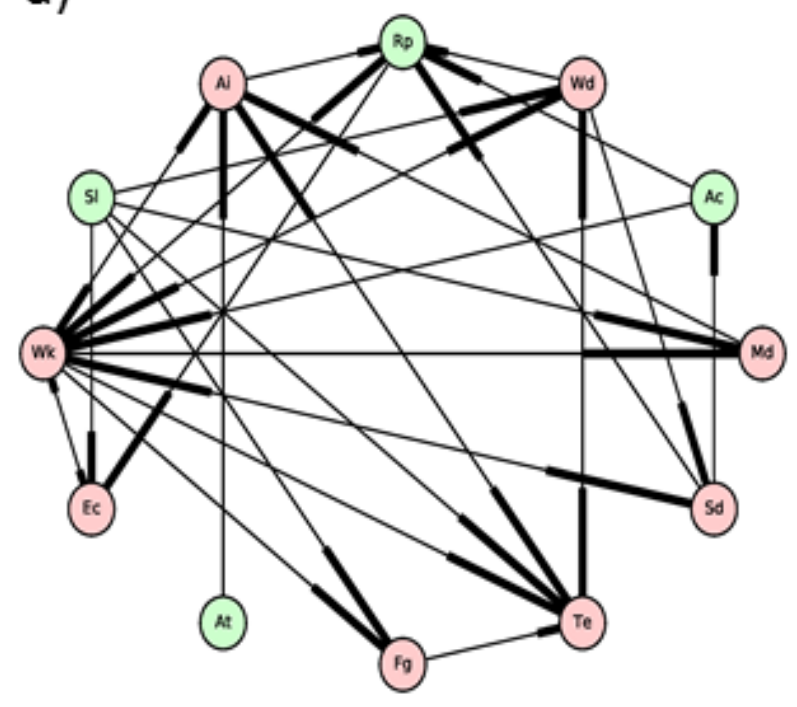

12: $A c^{+}, S d^{-} \rightarrow W k^{-}, R p^{-}$
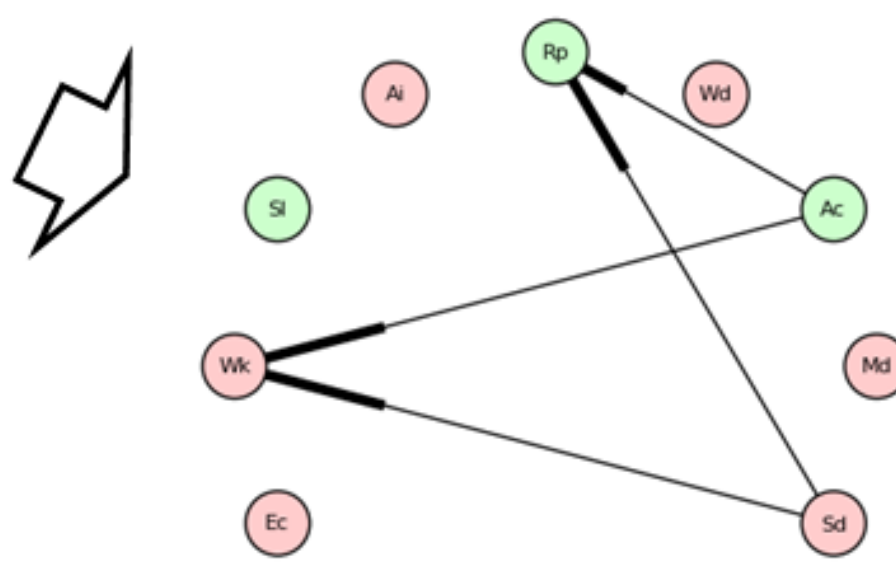

(A)

(T)

b)

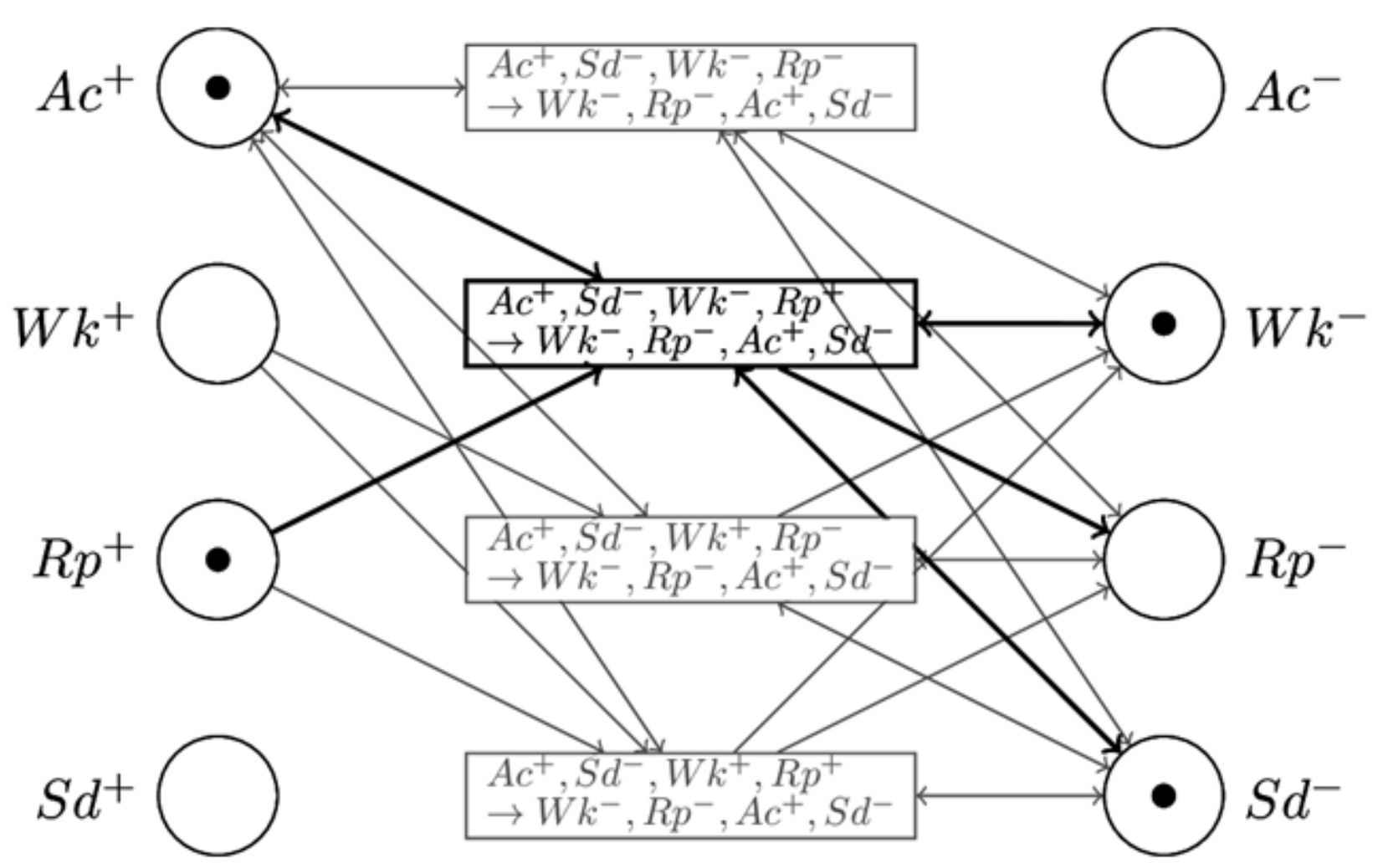

FIGURE 3 


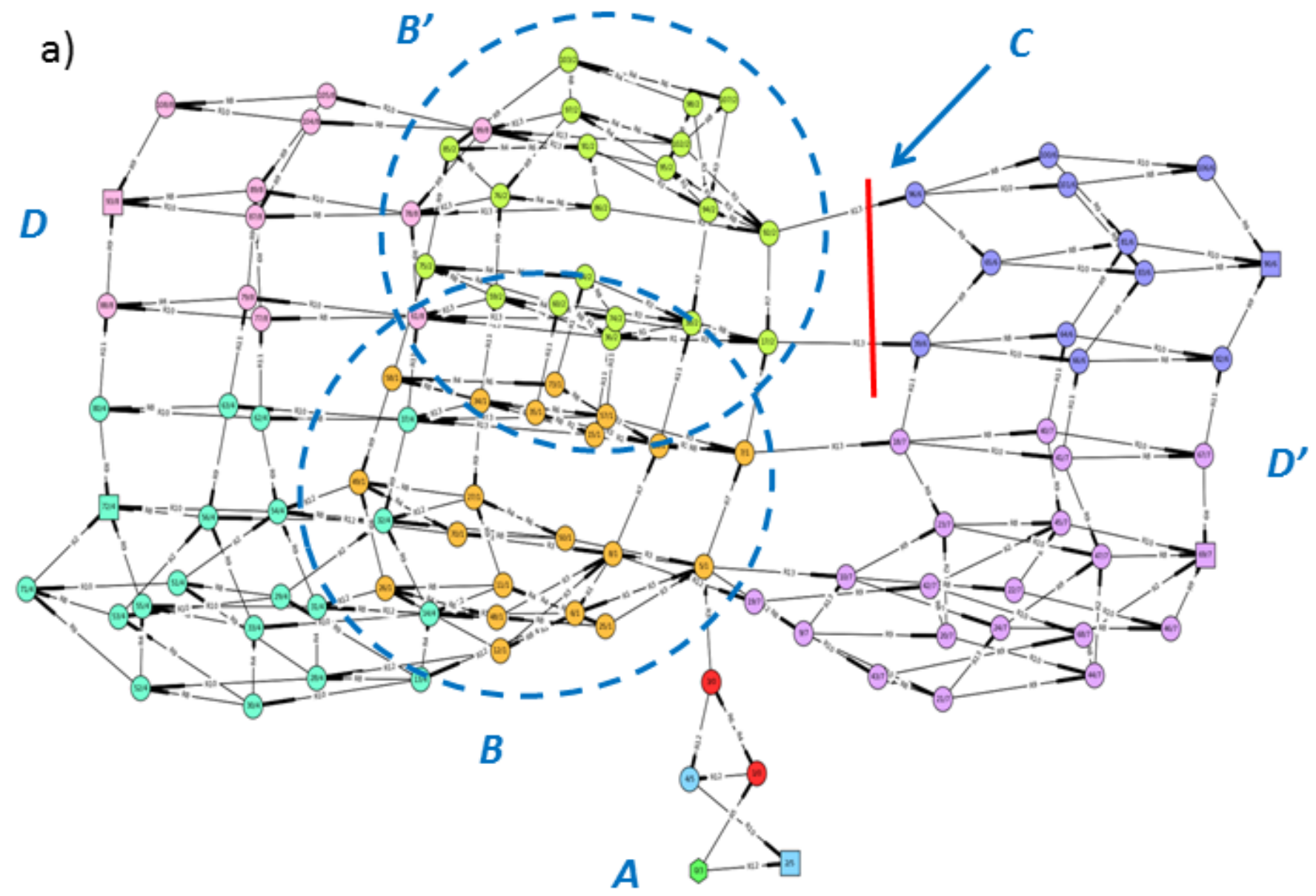

b)

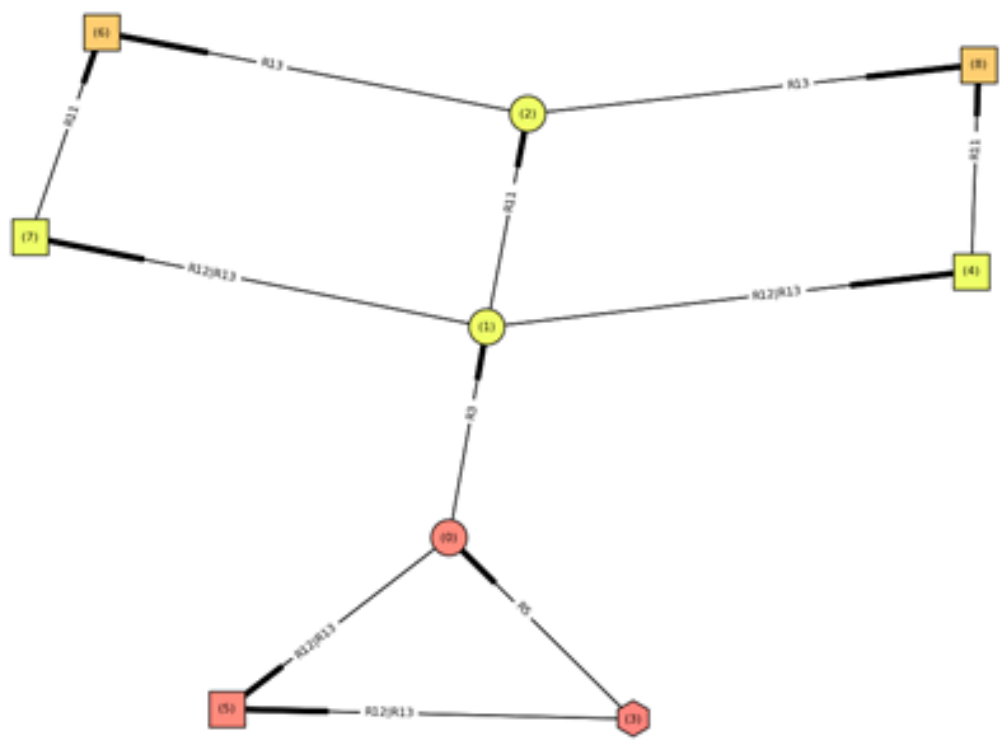

\section{FIGURE 4}

